\title{
GELATION IN COAGULATION AND FRAGMENTATION MODELS
}

\author{
M. Escobedo ${ }^{1}$, S. Mischler ${ }^{2,3}$ and B. Perthame ${ }^{3}$
}

\begin{abstract}
Rates of decay for the total mass of the solutions to Smoluchovski's equation with homogeneous kernels of degree $\lambda>1$ are proved. That implies that gelation always occurs. Morrey estimates from below and from above on solutions around the gelation time are also obtained which are in agreement with previously known formal results on the profile of solutions at gelling time. The same techniques are applied to the coagulation-fragmentation model for which gelation is established in some particular cases.
\end{abstract}

Key words: Smoluchowski's coagulation equation, decay rate, gelation, profile at gelling time, Morrey-Campanato norms, existence of solutions.

\section{Introduction and Main results}

The purpose of this work is to investigate gelling transition in coagulation and fragmentation model. The simplest model is the Smoluchowski coagulation equation describing irreversible aggregation processes between particles which coalesce and form larger and larger clusters.

Denoting by $f(t, y) \geq 0$ the density of clusters of size $y \in \mathbb{R}_{+}$at time $t \geq 0$, the continuous Smoluchowski's coagulation equation reads [32]

$$
\left\{\begin{aligned}
& \frac{\partial}{\partial t} f= Q_{c}(f)=\frac{1}{2} \int_{0}^{y} a\left(y-y^{\prime}, y^{\prime}\right) f\left(t, y-y^{\prime}\right) f\left(t, y^{\prime}\right) d y^{\prime} \\
&-f(t, y) \int_{0}^{\infty} a\left(y, y^{\prime}\right) f\left(t, y^{\prime}\right) d y^{\prime} \\
& f(0, y)=f_{i n}(y) \geq 0
\end{aligned}\right.
$$

We assume throughout this paper that the coagulation kernel $a$ is an homogeneous function of the form

$$
a\left(y, y^{\prime}\right)=\frac{\kappa}{2}\left(y^{\alpha} y^{\prime \beta}+y^{\beta} y^{\prime \alpha}\right) \quad \text { with } \quad 0 \leq \alpha \leq \beta \leq 1,
$$

\footnotetext{
${ }^{1}$ Departamento de Matemáticas, Universidad del País Vasco Apartado 644. Bilbao, Spain 48080 Email: mtpesmam@lg.ehu.es

${ }^{2}$ Laboratoire de Mathématiques Appliquées, Université de Versailles - Saint Quentin, 45 avenue des Etats-Unis, F-78035 Versailles. Email: mischler@math.uvsq.fr

${ }^{3}$ DMA, UNM 8553, Ecole Normale Supérieure, 45 rue d'Ulm, F-75230 Paris cedex 05 Email: perthame@dma.ens.fr
} 
where we may take $\kappa=1$ without any loss of generality. We refer to Remark 4.2 and Remark 7.1 for some extensions of our analysis to more general kernels. Since we are interested in gelling transition we only consider the case

$$
\lambda:=\alpha+\beta>1 .
$$

We refer to the review paper of D.J. Aldous [2] and D.L. Drake [11] for a basic physical description of coagulation-fragmentation models as well as many other references concerning physical motivations and mathematical analysis.

Let us emphasize that the results presented here are also valid for the discrete Smoluchowski coagulation equation [34]

$$
\left\{\begin{aligned}
\frac{\partial}{\partial t} c_{i} & =\frac{1}{2} \sum_{j=1}^{i-1} a(i-j, j) c_{i-j}(t) c_{j}(t)-c_{i}(t) \sum_{j=1}^{\infty} a(i, j) c_{j}(t), \\
c_{i}(0) & =c_{i, i n}, \quad \text { for any } i \in \mathbb{N}^{*},
\end{aligned}\right.
$$

where $c_{i}(t) \geq 0$ denotes the concentration of clusters of size $i \in \mathbb{N}^{*}$. Most of our results also extend (with minor modifications) to the non homogeneous continuous or discrete Smoluchowski coagulation equation. In order to avoid repetitions, we restrict our exposition mainly to the continuous homogeneous model (1.1). We quote the slight differences between the different models in section 8 below.

The key point in the analysis of equation (1.1) is the following identity: for every measurable functions $f$ and $\psi$ :

$$
\begin{aligned}
\int_{0}^{\infty} Q_{c}(f) \psi d y & =\frac{1}{2} \int_{0}^{\infty} \int_{0}^{\infty} a\left(y, y^{\prime}\right) f(y) f\left(y^{\prime}\right) \tilde{\psi}\left(y, y^{\prime}\right) d y d y^{\prime} \\
\tilde{\psi}\left(y, y^{\prime}\right) & =\psi\left(y+y^{\prime}\right)-\psi(y)-\psi\left(y^{\prime}\right) .
\end{aligned}
$$

This identity is obtained formally performing the change of variables $\left(y, y^{\prime}\right) \rightarrow$ $\left(z=y-y^{\prime}, y^{\prime}\right)$ in the first term of $Q_{c}(f)$ in (1.5) and is therefore rigorous when $f y^{\beta} \psi \in L^{1}\left(\mathbb{R}_{+}\right)$.

As an immediate consequence, when we choose $\psi(y)=y$ in (1.5), so that (1.6) vanishes, we get (at least formally) the conservation of mass

$$
\frac{d}{d t} \int_{0}^{\infty} y f(t, y) d y=0 .
$$

Under the assumption

$$
0 \leq f_{\text {in }} \in L_{1}^{1}\left(\mathbb{R}_{+}\right), \quad f_{\text {in }} \not \equiv 0
$$

it is well known that there exists a solution to (1.1) and (1.4) since the pionner works of Melzak [31], Sockmayer [42] and McLeod [29] [30] for the simplest 
cases $\alpha=\beta=0$. We also refer to [43], [28], [16], [5], [17] for the case $\alpha, \beta<$ 1 and to [14], [23] who revisited the case $\alpha=\beta=1$. The case $0<\alpha<$ $1, \beta=1$ is treated in Corollary 2.9 below. Exact solutions have also been constructed for special initial data (in particular for monodisperse distribution in the case of Smoluchovski's model) in [29], [28], [37], [27]. Finally, uniqueness (and existence) of solution has been proved in [21], [3], [39] and [13] under the assumption $\lambda \leq 1$, but uniqueness of solution is an open problem under the general assumption (1.2).

Here and below we use the notations $g \in L_{k}^{1}\left(\mathbb{R}_{+}\right)$to denote a measurable function such that

$$
\|g\|_{L_{k}^{1}}:=\int_{0}^{\infty}|g(y)|\left(1+y^{k}\right) d y<\infty
$$

and for such a function we define

$$
M_{k}(g):=\int_{0}^{\infty} g(y) y^{k} d y .
$$

The solutions to (1.1) obtained in the references quoted above always satisfy the following estimates

$$
\begin{gathered}
M_{0}\left(t_{1}\right) \leq M_{0}\left(t_{0}\right), \quad \text { (the number of particles decreases), } \\
M_{1}\left(t_{1}\right) \leq M_{1}\left(t_{0}\right), \quad \text { (the total mass decreases), }
\end{gathered}
$$

for any $t_{1} \geq t_{0} \geq 0$ (see [23] and Theorem 2.4).

One of the most relevant questions from the physical point of view, and mathematically interesting, is whether one has equality or strict inequality in (1.11). This is the gelation problem.

For $\lambda>1$, it is known that fo $\mathrm{r} f_{\text {in }} \in L_{\lambda}^{1}$ a solution of (1.1) can be constructed in such a way that it is mass conserving for small time (see [9] and [22]) i.e.:

$$
\exists T>0 \quad M_{1}(t):=M_{1}(f(t, .)) \equiv M_{1}(0) \quad \forall t \in[0, T] .
$$

For the discrete model (1.4) (then $M_{k} \leq M_{\ell}$ for $k \leq \ell$ ) and initial data satisfying $M_{2}(0)<\infty$, this fact can be easily understood using the following elementary argument. Thanks to (1.5)-(1.6) with $\psi(y)=y^{2}$, we have

$$
\frac{d}{d t} M_{2}(t)=M_{1+\alpha}(t) M_{1+\beta}(t) \leq M_{2}(t)^{2},
$$

and this differential inequality provides an (a priori) bound on the $L_{2}^{1}$ norm on a small interval $[0, T]$. The computation leading to (1.7) is therefore rigourous. 
Moreover, conservation of mass (1.12) holds true with $T=+\infty$ under the assumption $\lambda \leq 1$, see [43], [41]. For the discrete model (1.4) we argue as follows

$$
\begin{aligned}
\frac{d}{d t} M_{2}(t) & =M_{1+\alpha}(t) M_{1+\beta}(t) \\
& \leq M_{1}^{1-\alpha}(t) M_{2}^{\alpha}(t) M_{1}^{1-\beta}(t) M_{2}^{\beta}(t) \leq M_{1}(0) M_{2}(t),
\end{aligned}
$$

where we have used twice the Holder inequality and (1.11). That differential inequality provides an (a priori) bound on the $L_{2}^{1}$ norm of the solution on every bounded interval $[0, T]$.

However, for $\lambda>1$ mass conservation is expected to break down in finite time i.e.: there exists $T_{g} \in \mathbb{R}_{+}$, called gelation time, such that

$$
M_{1}(t) \equiv M_{1}(0) \quad \forall t<T_{g}, \quad M_{1}(t)<M_{1}(0) \quad \forall t>T_{g} .
$$

This fact was conjectured by Lushnikov and Ziff independently at the end of the 70 's.

This gelation phenomenon has been first adressed in the physical litterature, based on explicit solutions for special initial data ([28], [37], [27], [14]) and on formal scaling arguments [18].

The physical interpretation is that after gelation, some mass is lost under the form of a particle of infinite size $(y=\infty)$ with mass $M_{i n}-M_{1}(t)$, the so-called gel part. The particles of density $f(t, y)$ are then called the sol part. It is then a microscopic description of a phase transition.

The first universal and rigourous argument concluding to gelation seems to have been given by [28] in the case $\alpha=\beta=1$. More recently, using probability arguments, I. Jeon [20] was able to construct gelling solutions to (1.4) for any initial datum when $\lambda>1$. P. Laurençot [23] has obtained a decay rate for the total mass $M_{1}(t)$ in the particular case $\alpha=\beta=1$. We present here a sketch of the proof. Taking $\psi=1$ in (1.5)-(1.6) we get that a solution $f$ to (1.1) satisfies for any $t_{1} \geq t_{0} \geq 0$

$$
\frac{1}{2} \int_{t_{0}}^{t_{1}} \int_{0}^{\infty} \int_{0}^{\infty} a\left(y, y^{\prime}\right) f(t, y) f\left(t, y^{\prime}\right) d y d y^{\prime} d t=M_{0}\left(t_{0}\right)-M_{0}\left(t_{1}\right) .
$$

Thanks to the elementary inequality

$$
\left(y y^{\prime}\right)^{\lambda / 2} \leq\left(y^{\alpha} y^{\prime \beta}+y^{\beta} y^{\prime \alpha}\right) / 2
$$

and the positivity of $M_{0}$ we deduce the following first fundamental estimate

$$
\int_{t_{0}}^{\infty} M_{\lambda / 2}^{2}(t) d t \leq 2 M_{0}\left(t_{0}\right) \quad \forall t_{0} \geq 0
$$

Now, when $\alpha=\beta=1$ (so that $\lambda / 2=1$ ), we deduce from (1.16) and (1.11) that

$$
t M_{1}(t)^{2} \leq \int_{0}^{t} M_{1}^{2}(s) d s \leq 2 M_{0}(0) .
$$


This implies the following decay rate on the total mass $M_{1}(t)$ :

$$
M_{1}(t) \leq \frac{2 M_{0}(0)}{\sqrt{t}} \quad \forall t \geq 0 .
$$

In particular, $M_{1}(t)$ is not constant and gelation must occur in finite time.

Finally, for modified models, gelation and non existence of solutions (which may be considered as instantaneous and complete gelation) have been proved in [40], [4], [8], [6].

Our first main result states that for any weak solution $f$ (see Section 2 for a precise definition) to the coagulation equation, with homogeneous kernel $a$ given by $(1.2),(1.3)$, gelation occurs in finite time.

Theorem 1.1 Assume $f_{\text {in }} \not \equiv 0$. For every weak solutions $f$ to (1.1)-(1.3),(1.8) there exists a positive constant $C_{*}$ (depending on $\left.M_{0}(0), M_{1}(0), \lambda\right)$ such that for any $t \geq 0$

$$
M_{1}(t) \leq \frac{C_{*}}{(1+t)^{1 / \lambda}} .
$$

As a consequence, gelation occurs in finite time: (1.14) holds with the following upper bound on $T_{g}$ :

$$
T_{g} \leq T_{*}:=\left(\frac{C_{*}}{M_{1}(0)}\right)^{\lambda} .
$$

This result is a consequence of new momentum estimates (see Theorem 2.2 and Corollary 2.3). It recovers the previous result by I. Jeon [20] and extends it in several directions. First, our result is established for any weak solution and not for a particular well constructed solution (recall that uniqueness is not known). Our result also holds both for continuous and discrete models and is easy to extend to a non homogeneous setting (see Section 8). Finally, our proof is completely different from Jeon's proof (it is much more related to P. Laurençot's proof, [23]) and is very simple.

Once gelation is established, one may try to determine the asymptotic profile of the solution $f$ at gelling time $T_{g}$. Explicit exact solutions and formal arguments indicate that it should be a self similar profile, with algebraic spatial decay determined by the value of $\lambda$ in (1.2). More precisely:

$$
f\left(T_{g}, y\right) \sim y^{-\frac{3+\lambda}{2}} \text { when } y \rightarrow \infty
$$

see [18], [14], [10]. We present here a first rigorous result, in terms of MorreyCampanato type estimates, which hold for every weak solution, and which is in agreement with (1.20) although it is less precise.

Theorem 1.2 Let $f$ be any weak solution to (1.1)-(1.3) and (1.8). Then, for any $\tau>0$ and any $0 \leq T_{0} \leq T_{1}$ such that

$$
M_{1}\left(T_{1}\right)<M_{1}\left(T_{0}\right),
$$


$f$ satisfies:

$$
\forall R>0 \quad \sup _{S>R} \int_{T_{0}}^{T_{1}}\left(\frac{1}{S^{\tau}} \int_{0}^{S} f(t, y) y^{\lambda / 2+1 / 2+\tau} d y\right)^{2} d t \leq C_{1},
$$

and

$$
\forall R>0 \quad \int_{T_{0}}^{T_{1}} \sup _{S>R}\left(\frac{1}{S^{\tau}} \int_{0}^{S} f(t, y) y^{\lambda / 2+1 / 2+\tau} d y\right)^{2} d t \geq C_{1}^{-1},
$$

where $C_{1} \in(0, \infty)$ depends on $\alpha, \beta, f, T_{0}, T_{1}$ and $\tau$ but not on $R$.

The Morrey norms in (1.22) and (1.23) are classicaly used to include functions with a specific algebraic decay at infinity. The following Corollary relates the estimates (1.22) and (1.23) to the algebraic decay (1.20)

Corollary 1.3 Assume furthermore that $f$ has a spatial decay profile $\xi$, i.e.:

$$
\left\{\begin{array}{l}
\exists \Lambda, M \in \mathbb{R}_{+}, \exists \xi(y) \quad \text { s.t. } \quad \forall t \in\left[T_{0}, T_{1}\right], \forall y \geq M \\
\Lambda^{-1} \xi(y) \leq f(t, y) \leq \Lambda \xi(y) .
\end{array}\right.
$$

Then, the profile $\xi$ satisfies

$$
C_{2}^{-1} \leq \lim \sup \xi(y) y^{3 / 2+\lambda / 2}, \quad \liminf \xi(y) y^{3 / 2+\lambda / 2} \leq C_{2},
$$

for some $C_{2} \in \mathbb{R}_{+}$depending on $C_{1}, \Lambda$ and $T_{1}-T_{0}$. Moreover, if we also know that

$$
\xi(y)=y^{-\theta} \xi_{0}(y), \quad \text { with } \quad \xi_{0}(y) y^{-\kappa} \underset{y \rightarrow \infty}{\longrightarrow} 0 \forall \kappa>0,
$$

then $\theta=3 / 2+\lambda / 2$ and $\xi_{0} \equiv$ Const.

It is then clear from Theorem 1.2 and its Corollary that, at gelling time, the only possible algebraic decay for a solution is $3 / 2+\lambda / 2$. But since we are not able to prove (1.24) nor (1.26), other intrincated large size asymptotic behaviors are possible which could be in agreement with estimates (1.22) and (1.23).

We next investigate how the techniques that we have introduced for the coagulation equation can be used for the coagulation-fragmentation equation

$$
\frac{\partial}{\partial t} f=Q_{c}(f)+Q_{f}(f)
$$

with $Q_{c}$ is given by (1.1) and (1.2), and $Q_{f}$ is defined by

$$
Q_{f}(f)=-\frac{1}{2} f(y) \int_{0}^{y} b\left(y-y^{\prime}, y^{\prime}\right) d y^{\prime}+\int_{0}^{\infty} b\left(y, y^{\prime}\right) f\left(y+y^{\prime}\right) d y^{\prime}
$$

This equation takes into account not only the coagulation but also the fragmentation processes by which the clusters break apart into smaller peaces. We assume throughout this paper that the fragmentation kernel $b$ satisfies

$$
0 \leq b\left(y, y^{\prime}\right)=b\left(y^{\prime}, y\right) \leq B\left(y+y^{\prime}\right)
$$


with

$$
B(z)=\frac{B}{(1+z)^{\gamma}}
$$

for some $B>0$ and $\gamma$ satisfying

$$
\frac{\lambda}{2}+\gamma \geq \frac{3}{2}
$$

The key identity for the fragmentation term, which plays the same role as (1.5)-(1.6) for the coagulation term, is

$$
\begin{aligned}
\int_{0}^{\infty} Q_{f}(f) \psi d y & =-\frac{1}{2} \int_{0}^{\infty} \int_{0}^{\infty} b\left(y, y^{\prime}\right) f\left(y+y^{\prime}\right) \tilde{\psi}\left(y, y^{\prime}\right) d y d y^{\prime} \\
& =\frac{1}{2} \int_{0}^{\infty} f(z) k_{\psi}(z) d z
\end{aligned}
$$

with

$$
k_{\psi}(z)=\int_{0}^{z} b(y, z-y)(\psi(y)+\psi(z-y)-\psi(z)) d y
$$

for any $f \in L_{1}^{1}$ and any $\psi \in L^{\infty}$. As a consequence, the same formal conservation of mass (1.7) holds for the coagulation-fragmentation equation (1.27). Existence results of solutions to the Cauchy problem associated to (1.32) have been proved under different growth assumptions on the kinetic kernels $a$ and $b$ and we refer to [36], [3], [12], [7] for the discrete model and [31], [1], [38], [13], [23] and [26] for the continuous model. See also Corollary 2.9.

Concerning gelation phenomenon for the coagulation-fragmentation equation, rather few results are known. Notice that the two phenomena, coagulation and fragmentation, have opposite effects with respect to gelation. As we have already seen, if no fragmentation is present, and the coagulation is strong $(\lambda>1$ in (1.2)), then gelling transition occurs to all the weak solutions (that is Theorem 1.1). On the other hand, under the condition $\lambda \leq 1$, or if the fragmentation kernel $b$ is strong enough with respect to the coagulation kernel $a$, solutions exists which preserve the total mass for all the time, see [41], [7], [3], [15].

An illustrative example is the following. Consider the case $a\left(y, y^{\prime}\right)=y y^{\prime}$ and $b\left(y, y^{\prime}\right)=1$. Then using (1.5) and (1.32) with $\psi(y)=1$ and $\psi(y)=y^{2}$, we get thanks to the Cauchy-Schwarz inequality $M_{2}^{2} \leq M_{1} M_{3}$

$$
\frac{d}{d t} M_{0}=-\frac{1}{2} M_{1}^{2}+\frac{1}{2} M_{1}=\frac{1}{2}\left(1-M_{1}\right) M_{1}
$$

and

$$
\frac{d}{d t} M_{2}=\frac{1}{2} M_{2}^{2}-\frac{1}{2} M_{3} \leq \frac{1}{2}\left(M_{1}-\frac{1}{3}\right) M_{3} .
$$

On one hand, we see from (1.35) that if $M_{1}(0) \leq 1 / 3$ then $M_{2}$ is (formally) decreasing and then in this case one can build mass conserving solution. On the other hand, if $M_{1}(0)>1$ then from (1.34) any solution gels in finite time 
(otherwise $M_{1}(t)$ is a constant and $M_{0}(t)$ vanishes in finite time, which is a contradiction).

The question is then twofold. On the one hand, for which kernels $a$ and $b$ do the coagulation-fragmentation equation have gelling solutions? On the other hand, for which kernels $a$ and $b$ do all the solutions undergo gelling transition? We are far from answering these to questions, but Theorem (1.4) stated below gives some gelation criteria for the coagulation-fragmentation model and is a partial result in that direction.

Theorem 1.4 Assume $\lambda>1$ and $\gamma \geq 3 / 2-\lambda / 2$. Then, for any weak solution of the equation (1.27) gelation occurs when

$$
M_{1}(0) \text { is large enough; }
$$

or, without any condition on $M_{1}(0)$, when one of the two following conditions is satisfied

$$
b \text { has a compact support; }
$$

$$
\gamma>1 \text { and }\left\{\begin{array}{l}
a\left(y, y^{\prime}\right)=r(y)^{\alpha} r\left(y^{\prime}\right)+r(y) r\left(y^{\prime}\right)^{\alpha}, \alpha \in(0,1] \\
1 \leq r(y) \sim y \text { for large } y
\end{array}\right.
$$

Previous results on that direction have been proved by I. Jeon [20] and P. Laurençot [23]. To our knowledge Theorem 1.4 is the first result establishing systematic gelation for the complete coagulation-fragmentation equation (with $b \not \equiv 0)$. Notice that 1.38 includes the case of discrete coagulation-fragmentation equation with kernel $a(i, j)=i^{\alpha} j+i j^{\alpha}$. Notice also that when $\gamma>\frac{3}{2}-\frac{\lambda}{2}$, Theorem 1.2 may be applied and therefore the expected profile of the solution at gelling time is again like $y^{-\left(\frac{3}{2}+\frac{\lambda}{2}\right)}$.

The outcome of the rest of the paper is as follows:

Section 2. More about our results: moment estimates.

Section 3. Proof of the estimates from above for the coagulation equation.

Section 4. Gelation for the coagulation equation.

Section 5. Estimates from below and profile at gelling time for the coagulation equation.

Section 6. Behaviour of the solutions to the coagulation-fragmentation equation. Section 7. Existence result for the coagulation fragmentation equation.

Section8. Extensions to non homogeneous models.

\section{More about our results: moment estimates}

In this section we state in detail some new estimates which give rise, in particular to the main results stated in the introduction. For the sake of completeness we first recall the definition of weak solution to (1.1) and (1.27). 
Definition 2.1 We say that a function $f: \mathbb{R}_{+}^{2} \rightarrow \mathbb{R}_{+}$is a weak solution to the coagulation-fragmentation equation (1.27) if $f \in C\left([0, \infty) ; L^{1}\right) \cap L^{\infty}\left(0, T ; L_{1}^{1}\right)$ for any $T>0$,

$$
\forall t \geq 0 \quad M_{1}(t) \leq M_{1}(0)
$$

and for any $T_{1} \geq T_{0} \geq 0$ and any $\psi \in L^{\infty}\left(\mathbb{R}_{+}\right)$

$$
\begin{aligned}
& {\left[\int_{0}^{\infty} f(t, y) \psi(y) d y\right]_{T_{0}}^{T_{1}}=} \\
& =\frac{1}{2} \int_{T_{0}}^{T_{1}} \int_{0}^{\infty} \int_{0}^{\infty}\left(a f(t, y) f\left(t, y^{\prime}\right)-b f\left(t, y+y^{\prime}\right)\right) \tilde{\psi}\left(y, y^{\prime}\right) d y d y^{\prime} d t
\end{aligned}
$$

with a given by (1.2)-(1.3) and b satisfying (1.29)-(1.31). Solutions to the coagulation equation (1.1) are defined in the same way taking $b \equiv 0$.

It is possible to define the solutions of equation (1.1) and (1.27) in many other ways. For instance we may impose $f$ to be a solution of (1.1) or (1.27) in the distributional sense in $[0, T) \times \mathbb{R}_{+}$for any $T>0$ or in the mild sense. But anyway, as it is proved in [26], all these definitions are equivalent. Let us also emphasize that condition (2.1) is made in order to select a physically relevant solution. Nevertheless, it is not necessary to impose such an aditional condition for the kernels $a$ and $b$ that we are considering in this paper (except when $\gamma=3 / 2-\lambda / 2)$, since we shall prove that $M_{1}(t)$ is a decreasing function. Finally, we will not repeat anymore the assumptions on $a$ and $b$ which are, except if it is specified, those of the Definition 2.2.

Our first result is a family of a posteriori upper bounds on the weak solutions to the coagulation equation (1.1).

Theorem 2.2 For any increasing function $\Phi: \mathbb{R}_{+} \rightarrow \mathbb{R}_{+}$such that $\Phi(0)=0$ and

$$
C_{\Phi}:=\int_{0}^{\infty} \Phi^{\prime}(A) A^{-\frac{1}{2}} d A<\infty
$$

for any weak solution $f$ to (1.1), and for all $T \geq 0$, there holds

$$
\int_{T}^{\infty}\left(\int_{0}^{\infty} f(t, y) y^{\lambda / 2} \Phi(y) d y\right)^{2} d t \leq 2 C_{\Phi}^{2} M_{1}(T) .
$$

Under suitable choices of functions $\Phi$ we deduce the following refined moment estimates.

Corollary 2.3 For every weak solution $f$ to (1.1) and every $T \geq 0$, there holds:

$$
\int_{T}^{\infty}\left(\int_{R}^{\infty} f(t, y) y d y\right)^{2} d t \leq C_{\lambda} R^{1-\lambda} M_{1}(T)
$$


for any $R>0, \lambda \in(1,2)$;

$$
\int_{T}^{\infty}\left(\frac{1}{R^{\tau}} \int_{0}^{R} f(t, y) y^{\lambda / 2+1 / 2+\tau} d y\right)^{2} d t \leq C_{\tau} M_{1}(T)
$$

for any $R, \tau>0$;

$$
\int_{T}^{\infty}\left(\int_{e}^{\infty} f(t, y) \frac{y^{\lambda / 2+1 / 2}}{(\ln y)^{\delta}} d y\right)^{2} d t \leq C_{\delta} M_{1}(T)
$$

for any $\delta>1$;

$$
\int_{T}^{\infty} M_{k}^{2}(t) d t \leq C_{k}\left(M_{0}(T)+M_{1}(T)\right),
$$

for any $k \in[\lambda / 2, \lambda / 2+1 / 2)$ if $\lambda \in(1,2)$ and for $k=1$ if $\lambda=2$.

In particular, we see from $(2.8)$ that $M_{1} \in L^{2}\left(\mathbb{R}_{+}\right)$so that $M_{1}(t)$ can not be constant in time and gelation must occurs. In fact, rates of decay for the total mass $M_{1}(t)$ of any weak solution as stated in Theorem 1.1 can be deduced from (2.6) and the following result.

Theorem 2.4 Any weak solution $f$ to (1.1) satisfies

$$
M_{k} \text { is decreasing and } M_{k}(t) \rightarrow 0 \text { when } t \rightarrow \infty
$$

for any $k \in[0,1]$. Moreover, $M_{k}(t)$ is continuous for any $k \in[0,1)$ and $M_{1}(t)$ is right continuous.

We do not know whether (1.18) gives the generic decay of weak solutions for general nonnegative initial data $f_{\text {in }} \in L_{1}^{1}\left(\mathbb{R}^{+}\right)$. Nevertheless it may be improved in some cases. This is done in the following Corollary which has to be compared with [27] and [14].

Corollary 2.5 For any weak solution of the discrete coagulation equation (1.4) we have

$$
M_{1}(t) \leq \frac{C}{1+t} \text { for any } t \geq 0 .
$$

For any weak solution to (1.1) with initial data such that $M_{-q}(0)<\infty, q \geq 0$ :

$$
M_{1}(t) \leq \frac{C_{q}}{(1+t)^{\nu}} \text { for any } t \geq 0, \text { with } \nu=\frac{1}{1+\frac{\lambda-1}{1+q}} .
$$

For any weak solution to (1.1) with initial data such that $f_{\text {in }}(y)=0$ for $y \in[0, \delta]$ and some $\delta>0$,

$$
M_{1}(t) \leq \frac{C}{1+t} \text { for any } t \geq 0 .
$$


Notice that, thanks to (2.10) we recover the upper bound on the gelling time obtained in [20], namely $T_{g} \leq C / M_{1}(0)$.

We now present the lower bounds that we can derive on solutions around the gelling time.

Theorem 2.6 Let $f$ be solution of (1.1). Assume that

$$
\Delta M_{1}:=M_{1}\left(T_{0}\right)-M_{1}\left(T_{1}\right)>0 \text { for some } T_{0}<T_{1} .
$$

Then for any $R, \tau>0$ there exists $C_{\tau}$ such that

$$
\int_{T_{0}}^{T_{1}}\left(\sup _{S>R} \frac{1}{S^{\tau}} \int_{0}^{S} y^{\lambda / 2+1 / 2+\tau} f(t, y) d y\right)^{2} d t \geq C_{\tau} \Delta M_{1}
$$

and

$$
\int_{T_{0}}^{T_{1}}\left(\int_{e}^{\infty} \frac{y^{\lambda / 2+1 / 2}}{(\ln y)^{1 / 2}} f(t, y) d y\right)^{2} d t=+\infty
$$

As a consequence, combining (2.6) and (2.14) we obtain Theorem 1.2.

Let us quote some questions of interest.

\section{Open questions.}

1. Is $M_{1}(t)$ continuous?

2. At gelling time is the profile of $f$ exactly (1.20)?

3. Another way to understand the gelation phenomenon is to prove that for some $k>1$ and $0<T_{c}(k)<\infty$,

$$
\lim _{t \rightarrow T_{c}(k)} M_{k}(t)=\infty .
$$

For instance, when $\alpha=\beta=1$, (1.13) gives

$$
\frac{d}{d t} M_{2}(t)=M_{2}(t)^{2},
$$

which readily implies that $M_{2}(t)$ blows up in finite time and $T_{c}(2)=M_{2}(0)^{-1}$. We refer to [9] where it is shown, for the discrete model (1.4), that $M_{\lambda}(t)$ blows up in finite time for the coagulation kernel (1.2) with $\alpha \in(0,1), \beta=1$. It is in general an open problem to prove whether $T_{g}=T_{c}(k)$. Nevertheless, it is easy to check that in the simple case where $a\left(y, y^{\prime}\right)=y y^{\prime}$ we have $T_{g}=T_{c}(2)$, see [33] Theorem 2.2 page 411.

4. Does $T_{g}$ satisfies: $\forall t_{0}, t_{1}$ such that $t_{0}<T_{g} \leq t_{1}$,

$$
\liminf _{y \rightarrow \infty} f\left(t_{0}, y\right) y^{3 / 2+\lambda / 2}=0, \quad \liminf _{y \rightarrow \infty} f\left(t_{1}, y\right) y^{3 / 2+\lambda / 2}>0 ?
$$


We next investigate how the previous results may be extended to the coagulationfragmentation equation (1.27). We start with the extension of Theorem 2.2 and Corollary 2.3.

Theorem 2.7 Let $\Phi: \mathbb{R}_{+} \rightarrow \mathbb{R}_{+}$be an increasing function satisfying $\Phi(0)=0$ and (2.3). For any weak solution $f$ to (1.27) there holds

$$
\int_{t_{0}}^{t_{1}}\left(\int_{0}^{\infty} f(t, y) y^{\lambda / 2} \Phi(y) d y\right)^{2} d t \leq C_{\Phi}^{2}\left(4 M_{1}\left(t_{0}\right)+B^{2}\left(t_{1}-t_{0}\right)\right)
$$

for every $t_{1} \geq t_{0} \geq 0$. In particular, for convenient choices of $\Phi$, we obtain that for any $T \geq 0$ :

$$
\int_{0}^{T}\left(\frac{1}{R^{\tau}} \int_{0}^{R} f(t, y) y^{\lambda / 2+1 / 2+\tau} d y\right)^{2} d t \leq C_{\tau}\left(M_{1}(0)+B^{2} T\right)
$$

for any $R, \tau>0$;

$$
\int_{0}^{T}\left(\int_{e}^{\infty} f(t, y) \frac{y^{\lambda / 2+1 / 2}}{(\ln y)^{\delta}} d y\right)^{2} d t \leq C_{\delta}\left(M_{1}(0)+B^{2} T\right)
$$

for any $\delta>1$;

$$
\int_{0}^{T} M_{k}^{2}(t) d t \leq C_{k}\left(M_{0}(0)+M_{1}(0)+B^{2} T\right)
$$

for any $k \in[\lambda / 2, \lambda / 2+1 / 2)$ if $\lambda \in(1,2)$ and for $k=1$ if $\lambda=2$.

The ocurrence of gelation for large initial mass stated in Theorem 1.4 is a consequence of estimate (2.21) with $k=1$. The two other statements of Theorem 1.4 come from "variations around" the proof of Theorem 2.7.

Another consequence of Theorem (2.7), more precisely of the estimate (2.20), is the following existence result for equation (1.27). We notice that it is only new for the cases $\alpha \in(0,1)$ and $\beta=1$.

Corollary 2.8 Assume $\alpha \in(0,1)$ and $\beta=1$. For any initial datum $f_{i n} \in$ $L_{1}^{1}\left(\mathbb{R}_{+}\right)$, there exists a weak solution $f \geq 0$ to the coagulation-fragmentation equation (1.27) satisfying (2.1).

Let us finally state the following result about the profile at gelation time for the solutions to the coagulation-fragmentation equation (1.27).

Corollary 2.9 Assume that $\lambda / 2+\gamma>3 / 2$. Any weak solution to the coagulationfragmentation equation (1.27) satisfies $M_{1}(t)$ is decreasing and right continuous, and moreover, if (2.13) holds, then (2.14) and (2.15) also hold. 
As a consequence, gathering (2.19) in Theorem 2.7 and Corollary 2.9 we see that Theorem 1.2 (and hence Corollary 1.3) is also valid when $\lambda \in(1,2]$ and $\lambda / 2+\gamma>3 / 2$. Therefore, the only algebraic decay at gelling time may be $3 / 2+\lambda / 2$, as for the pure coagulation model.

Under the conditions of Theorem 1.4, any solution of the coagulation-fragmentation equation satisfies $(2.20),(2.21)$, just like those of the coagulation equation. In particular, their only possible asymptotic profile at $T_{g}$ with algebraic behaviour $y^{-\theta}$ as $y \rightarrow \infty$ is again $\theta=3 / 2+\lambda / 2$. This seems to indicate that, under these hypothesis on the coagulation and fragmentation kernels, the gelation of the solutions to the coagulation-fragmentation equation is dominated by the coagulation, and the fragmentation is only a small perturbation.

Open Problem 2.10 It is possible in some cases to use the same formal arguments as Ernst, Ziff and Hendricks in [14] to get some insight on the behaviour of the solutions to the coagulation fragmentation equation. Let us assume in what follows that a is given by (1.2) and that $b\left(y, y^{\prime}\right)=B\left(y+y^{\prime}\right)$ with $B$ given by (1.30). by

The loss of mass from smaller clusters with $y<L$ to larger clusters is given

$$
\frac{d}{d t} \int_{0}^{L} y f(t, y) d y=\int_{0}^{L} y Q(f) d y
$$

with

$$
\begin{aligned}
\int_{0}^{L} y Q(f) d y=\quad & -\int_{0}^{L} \int_{L-y}^{\infty} y f(t, y) f\left(t, y^{\prime}\right) a\left(y, y^{\prime}\right) d y d y^{\prime} \\
& +\int_{0}^{L} \int_{L-y}^{\infty} y f\left(t, y+y^{\prime}\right) b\left(y, y^{\prime}\right) d y^{\prime} d y
\end{aligned}
$$

Assume that at $T_{g}$ the solution is given by a pure power-law $f\left(T_{g}, y\right) \equiv C y^{-r}$. Then, if $r$ satisfies

$$
\max \{\beta+1,2-\gamma\}<r<\alpha+2
$$

we obtain

$$
\int_{0}^{L} y Q(f) d y=-K_{1} C^{2} L^{3+2 \lambda-2 r}+K_{2} C L^{3-\gamma-r}
$$

for some positive constants $K_{1}=K_{1}(\alpha, \beta, r)$ and $K_{2}=K_{2}(\gamma, r)$.

On the other hand we formally deduce from 2.22 that there is not conservation of mass if and only if:

$$
\lim _{L \rightarrow \infty} \int_{0}^{L} y Q(f) d y<0 .
$$

From 2.25 this only holds whenever $r \leq \min (3 / 2+\lambda / 2, \lambda+\gamma)$. Arguing now byy analogy with the formal argument used for the pure coagulation equation we then consider

$$
r=\min (3 / 2+\lambda / 2, \lambda+\gamma)
$$


We obtain then:

(i) If $\gamma+\lambda / 2>3 / 2$, so that $r=3 / 2+\lambda / 2$. Then,

$$
\int_{0}^{L} y Q(f) d y=-K_{1} C^{2}+K_{2} C L^{3 / 2-\lambda / 2-\gamma} \rightarrow-K_{1} C^{2}<0, \quad \text { as } \quad L \rightarrow \infty .
$$

Therefore, (2.26) holds for any $C>0$.

(ii) If $\gamma+\lambda / 2 \leq 3 / 2$, then $r=\lambda+\gamma$ and

$$
\int_{0}^{L} y Q(f) d y=\left(K_{2} C-K_{1} C^{2}\right) L^{3-\lambda-2 \gamma} .
$$

Therefore, (2.26) holds if and only if $C>\frac{K_{2}}{K_{1}}$, i.e. the initial datum is suffciently large.

On the ground of the above remarks we are lead to the following conjectures.

1.- If $\gamma+\lambda / 2>3 / 2$ : gelation occurs for all initial data and the gelling profile is given by $y^{-(3 / 2+\lambda / 2)}$. Moreover $M_{1}(t) \rightarrow 0$ as $t \rightarrow \infty$.

2.- If $\gamma+\lambda / 2 \leq 3 / 2$ and $\lambda+\gamma \geq 2$ there exists a positive constant $M_{1}^{*}$ such that:

If $M_{1}(0)>M_{1}^{*}$ gelation occurs, the gelling profile is like $y^{-(\lambda+\gamma)}$ and $M_{1}(t) \rightarrow M_{1}^{*}$ as $t \rightarrow \infty$.

If $M_{1}(0)<\leq M_{1}^{*}$ the mass of the solution is conserved for all the time.

Notice that when $\lambda+\gamma<2$ it is possible to construct global mass preserving solutions, see [7], [15].

\section{$3 \quad$ Upper bounds}

This section is devoted to the proof of the a posteriori estimates in Theorem 2.2 and Corollary 2.3.

Proof of Theorem 2.2. Consider a weak solution $f$ of equation (1.1). Choosing $\psi=\psi_{A}(y)=y \wedge A$ (which belongs to $L^{\infty}$ ) in (1.5) we get

$$
\begin{gathered}
\frac{1}{2} \int_{t_{0}}^{t_{1}} \int_{0}^{\infty} \int_{0}^{\infty} a\left(y, y^{\prime}\right) f(t, y) f\left(t, y^{\prime}\right)\left(-\tilde{\psi}_{A}\left(y, y^{\prime}\right)\right) d y d y^{\prime} d t= \\
\quad=\int_{0}^{\infty} f\left(t_{0}, y\right) \psi_{A} d y-\int_{0}^{\infty} f\left(t_{1}, y\right) \psi_{A} d y
\end{gathered}
$$

We compute

$(3.2)-\tilde{\psi}_{A}\left(y, y^{\prime}\right)=\left\{\begin{array}{lll}0 & \text { on } & \left\{y, y^{\prime} ; y+y^{\prime} \leq A\right\} \\ y+y^{\prime}-A & \text { on } & T_{A} \\ y & \text { on } & \left\{y, y^{\prime} ; y \leq A, y^{\prime} \geq A\right\} \\ y^{\prime} & \text { on } & \left\{y, y^{\prime} ; y \geq A, y^{\prime} \leq A\right\} \\ A & \text { on } & \left\{y, y^{\prime} ; y \geq A, y^{\prime} \geq A\right\},\end{array}\right.$ 
with

$$
T_{A}=\left\{y, y^{\prime} ; y \leq A, y^{\prime} \leq A, y+y^{\prime} \geq A\right\}
$$

From (1.15) and $-\tilde{\psi}_{A}\left(y, y^{\prime}\right) \geq 0$, and keeping only the term coming from the region $\left\{y \geq A, y^{\prime} \geq A\right\}$ in the collision integral (3.1), we deduce

$$
\int_{t_{0}}^{t_{1}}\left(\int_{A}^{\infty} y^{\lambda / 2} f(t, y) d y\right)^{2} d t \leq 2 \frac{M_{1}\left(t_{0}\right)}{A} \quad \forall A>0 .
$$

First, using Fubini's Theorem, we have

$$
\int_{0}^{\infty} f(t, y) y^{\lambda / 2} \Phi(y) d y=\int_{0}^{\infty} \Phi^{\prime}(A) \int_{A}^{\infty} f(t, y) y^{\lambda / 2} d y d A
$$

Next, using Cauchy-Schwarz inequality and Fubini's Theorem, we deduce from (3.4) and (3.5)

$$
\begin{aligned}
& \int_{t_{0}}^{t_{1}}\left(\int_{0}^{\infty} \Phi^{\prime}(A) \int_{A}^{\infty} f(t, y) y^{\lambda / 2} d y d A\right)^{2} d t \leq \\
& \quad \leq \int_{t_{0}}^{t_{1}} \int_{0}^{\infty} \frac{\Phi^{\prime}(A)}{A^{\frac{1}{2}}} d A \int_{0}^{\infty} \Phi^{\prime}(A) A^{\frac{1}{2}}\left(\int_{A}^{\infty} f(t, y) y^{\lambda / 2} d y\right)^{2} d A d t \\
& \quad \leq C_{\Phi} \int_{0}^{\infty} \Phi^{\prime}(A) A^{\frac{1}{2}} \int_{t_{0}}^{t_{1}}\left(\int_{A}^{\infty} f(t, y) y^{\lambda / 2} d y\right)^{2} d t d A \\
& \leq C_{\Phi} \int_{0}^{\infty} 2 M_{1}(t) \frac{\Phi^{\prime}(A)}{A^{\frac{1}{2}}} d A=2 C_{\Phi}^{2} M_{1}\left(t_{0}\right) .
\end{aligned}
$$

Theorem 2.2 then follows from (3.5) and (3.6) letting $t_{1} \rightarrow \infty$.

Proof of Corollary 2.3.

- Proof of (2.5). Taking $\Phi(y)=\left(y^{1-\lambda / 2}-(R / 2)^{1-\lambda / 2}\right)^{+}$, the constant $C_{\Phi}$ in $(3.6)$ is

$$
C_{\Phi}=\frac{2-\lambda}{\lambda-1}\left(\frac{R}{2}\right)^{\frac{1}{2}-\frac{\lambda}{2}}
$$

From (3.7) and (2.4) we get

$$
\int_{t_{0}}^{\infty}\left(\int_{R}^{\infty} f(t, y) y^{\lambda / 2}\left(\frac{y}{2}\right)^{1-\lambda / 2} d y\right)^{2} d t \leq C M_{1}\left(t_{0}\right) R^{1-\lambda}
$$

and (2.5) readily follows.

-Proof of (2.7). We define $\Phi(y)=\left(y^{1 / 2} /(\ln y)^{\delta}-r^{1 / 2} /(\ln r)^{\delta}\right)^{+}$with $\delta>1$ and $r=\exp (2 \delta)$. We easily verify that $\Phi$ is increasing and that the associated constant $C_{\Phi}$ by (3.6) is finite. We conclude using estimate (2.4) for this choice of $\Phi$ and the estimate $(2.5)$ with $R=e$. 
- Proof of (2.6). Taking $\Phi(y)=(y \wedge R)^{\frac{1}{2}+\tau}$, we get

$$
C_{\Phi}=\left(1+\frac{1}{2 \tau}\right) R^{\tau}
$$

and conclude.

- Proof of (2.8). For $k \in[\lambda / 2, \lambda / 2+1 / 2)$ we have for some constant $C_{i}$ depending on $\lambda$ and $k$

$$
\begin{aligned}
M_{k}^{2}(t) & \leq C_{1}\left(\int_{0}^{e} y^{\frac{\lambda}{2}} f(t, y) d y+\int_{e}^{\infty} y^{k} f(t, y) d y\right)^{2} \\
& \leq C_{2}\left[M_{\lambda / 2}^{2}(t)+\left(\int_{e}^{\infty} \frac{y^{\frac{\lambda}{2}+\frac{1}{2}}}{(\ln y)^{2}} f(t, y) d y\right)^{2}\right] .
\end{aligned}
$$

Then (2.8) follows from (1.16) and (2.7).

\section{Gelation}

This section is devoted to the proof of Theorem 1.1, Theorem 2.4 and Corollary 2.5.

Proof of (2.9) in Theorem 2.4. Define $\psi_{A}(y)=y^{k} \wedge A$ with $k \in[0,1]$ and $\tilde{\psi}_{A}\left(y, y^{\prime}\right)$ by (1.6). From the two following elementary inequalities

$$
(u \wedge A)+(v \wedge A) \geq(u+v) \wedge A \text { and } X^{k}+Y^{k} \geq(X+Y)^{k}
$$

and the fact that $z \mapsto z \wedge A$ is increasing, we get

$$
y^{k} \wedge A+y^{\prime k} \wedge A \geq\left(y^{k}+y^{\prime k}\right) \wedge A \geq\left(y+y^{\prime}\right)^{k} \wedge A,
$$

so that $\tilde{\psi}_{A} \geq 0$. For $t_{1} \geq t_{0} \geq 0$, we then deduce from the fundamental identity (1.5) that

$$
\int_{0}^{\infty} f\left(t_{1}, y\right) \varphi_{A}(y) d y \leq \int_{0}^{\infty} f\left(t_{0}, y\right) \varphi_{A}(y) d y \leq \int_{0}^{\infty} f\left(t_{0}, y\right) y^{k} d y .
$$

We conclude by Fatou Lemma (letting $A \rightarrow \infty$ ) that $t \mapsto M_{k}(t)$ is a decreasing function.

Proof of Theorem 1.1. We just need to prove (1.18). Our proof is based on the method introduced in [23] and on the new bound (2.5).

For given $R>0$ we have

$$
M_{1}^{2}(t) \leq 2\left(\int_{0}^{R} y f(t, y) d y\right)^{2}+2\left(\int_{R}^{\infty} y f(t, y) d y\right)^{2}
$$


and

$$
\left(\int_{0}^{R} y f(t, y) d y\right)^{2} \leq R^{2-\lambda} M_{\lambda / 2}^{2}(t)
$$

Gathering (4.1), (4.2) with (1.16) and (2.5) we get for any $R>0$

$$
\int_{T}^{\infty} M_{1}^{2}(t) d t \leq 4 R^{2-\lambda} M_{0}(T)+C_{\lambda} R^{1-\lambda} M_{1}(T) .
$$

Then, making the choice $R=M_{1}(T) / M_{0}(T)$ and using (1.10) we obtain

$$
\int_{T}^{\infty} M_{1}^{2}(t) d s \leq C_{\lambda} M_{0}(0)^{\lambda-1} M_{1}(T)^{2-\lambda}
$$

The decay rate (1.18) follows then from (4.4) and Lemma 4.1 below.

Lemma 4.1 Assume that the square integrable and decreasing function $M_{1}(t)$ satisfies

$$
\forall T \geq 0 \quad \int_{T}^{\infty} M_{1}^{2}(s) d s \leq C_{1} M_{1}(T)^{\theta},
$$

for some constants $C_{1}>0$ and $\theta \in(0,2)$. Then

$$
\forall t>0 \quad M_{1}(t) \leq C_{2} t^{-\frac{1}{2-\theta}},
$$

for some constant $C_{2}=C_{2}\left(C_{1}, \theta,\left\|M_{1}\right\|_{L^{2}}\right)>0$.

Proof of Lemma 4.1. The proof of Lemma 4.1 is classical. Nevertheless, for the sake of completeness, we present it here. Define

$$
u(t):=\int_{t}^{\infty} M_{1}^{2}(s) d s .
$$

We deduce from (4.5) that $u$ satisfies $\frac{d u}{d t} \leq-\left(\frac{u(t)}{C_{1}}\right)^{2 / \theta}$ and thus

$$
u(t) \leq\left(\frac{t}{C_{1}^{2 / \theta}}+\frac{1}{u(0)^{2 / \theta-1}}\right)^{-\frac{1}{2 / \theta-1}} .
$$

Since $M_{1}(t)$ is decreasing we also have

$$
\frac{t}{2} M_{1}^{2}(t) \leq \int_{t / 2}^{t} M_{1}^{2}(s) d s \leq u(t / 2),
$$

and Lemma 4.1 follows gathering (4.7) and (4.8).

End of the Proof of Theorem 2.4. We proceed in two steps. 
Step 1. For any $k \in[0,1)$ we prove that $M_{k}(t) \rightarrow 0$. Chosing $\psi(y)=\mathbf{1}_{[0, \rho]}(y)$ we find that $\tilde{\psi} \leq 0$ and therefore

$$
\int_{0}^{\rho} f(t, y) d y \leq \int_{0}^{\rho} f_{i n}(y) d y \text { for any } t \geq 0 .
$$

We deduce that, for any $k \in[0,1)$ and $\rho \in(0,1)$,

$$
\begin{aligned}
M_{k}(t) & \leq \rho^{k} \int_{0}^{\rho} f(t, y) d y+\frac{1}{\rho^{1-k}} \int_{\rho}^{\infty} y f(t, y) d y \\
& \leq \int_{0}^{\rho} f_{\text {in }}(y) d y+\frac{1}{\rho^{1-k}} M_{1}(t)
\end{aligned}
$$

and the right hand side term goes to 0 when $t \rightarrow \infty$ and $\rho \rightarrow 0$.

Step 2. For any $k \in[0,1)$ we prove that $M_{k}(t)$ is continuous and $M_{1}(t)$ is right continuous. Since by definition $f \in C\left([0, \infty) ; L^{1}\left(\mathbb{R}_{+}\right)\right) \cap L^{\infty}\left(0, T ; L_{1}^{1}\left(\mathbb{R}_{+}\right)\right)$for all $T>0$, it readily follows that $M_{k}(t)$ is continuous for any $k \in[0,1)$. Moreover, for any $t_{0} \leq t_{1}$ we have

$$
\begin{gathered}
0 \leq M_{1}\left(t_{0}\right)-M_{1}\left(t_{1}\right)=\int_{0}^{\infty} f\left(t_{0}, y\right) y \wedge A d y-\int_{0}^{\infty} f\left(t_{1}, y\right) y \wedge A d y \\
\quad+\int_{0}^{\infty} f\left(t_{0}, y\right)(y-A)^{+} d y-\int_{0}^{\infty} f\left(t_{1}, y\right)(y-A)^{+} d y \\
\leq \quad \int_{t_{0}}^{t_{1}} \int_{0}^{\infty} \int_{0}^{\infty} a f(t, y) f\left(t, y^{\prime}\right)\left(-\tilde{\psi}_{A}\right) d y d y^{\prime} d t+\int_{0}^{\infty} f\left(t_{0}, y\right)(y-A)^{+} d y .
\end{gathered}
$$

For fixed $t_{0} \geq 0$ we fix $A$ large enough so that the second term is small, and then $t_{1}$ close enough to $t_{0}$, so that the first term is small. This exactly means that $M_{1}(t)$ is right continuous.

Proof of Corollary 2.5. Here again we follow [23].

Proof of 2.10 and 2.12. For the discrete coagulation equation we have

$$
M_{0}(t) \leq C M_{1}(t) .
$$

with $C=1$. For the continuous coagulation equation with vanishing initial data near the origin, we use 4.9 with $\rho=\delta$ which implies that $f(t, y) \equiv 0$ for any time $t \geq 0$ and every $y \in[0, \delta]$. Therefore (4.11) also holds with $C=1 / \delta$. In both cases, (4.4) and (4.11) imply

$$
\int_{T}^{\infty} M_{1}^{2}(t) d t \leq C_{\lambda} M_{1}(T) .
$$

The decay rate (2.10) then follows again from Lemma 4.1. 
Proof of 2.11. Under the assumption $M_{-q}(0)<\infty$ we may write

$$
M_{0}(t) \leq R^{q} M_{-q}(0)+\frac{1}{R} M_{1}(t) .
$$

Gathering (4.3) and (4.12) we have

$$
\begin{aligned}
\int_{T}^{\infty} M_{1}^{2}(t) d t & \leq C_{\lambda}\left(R^{2-\lambda} M_{0}(T)+R^{1-\lambda} M_{1}(T)\right) \\
& \leq C_{\lambda}\left(R^{2-\lambda+q} M_{-q}(0)+R^{1-\lambda} M_{1}(T)\right) \\
& \leq C_{\lambda}\left(1+M_{-q}(0)\right) M_{1}(T)^{1+\frac{1-\lambda}{1+q}}
\end{aligned}
$$

with the choice $R^{1+q}=M_{1}(T)$. We conclude again using Lemma 4.1.

Remark 4.2 One can also prove that gelation occurs for any coagulation kernel satisfying the assumption

$$
a>0 \text { on } \mathbb{R}_{+}^{2} \quad \text { and } \quad a \geq a_{0} \text { on }\left[A_{0}, \infty\right)^{2}
$$

with $a_{0}$ of the shape (1.2) and $A_{0}$ large. Indeed, in this case we have for any $A_{1}>0$

$$
a \geq \kappa_{A_{1}} a_{0} \text { on }\left[A_{1}, \infty\right)^{2},
$$

with $\kappa_{A_{1}}>0$. We then proceed as in the proof of Theorem 1.4 step 2 in Section 6, choosing $A_{1}$ small enough in (6.9) and (6.10).

\section{Estimates from below and profile at gelling time}

This section is devoted to the proof of Theorem 2.6, Theorem 1.2 and Corollary 1.3 .

Proof of Theorem 2.6. Step 1. Preliminaries. Let put again $\psi_{A}(y)=y \wedge A$. We deduce from (3.1), (3.2) and assumption (2.13) that there exists $A_{0} \geq 0$ such that

$$
\forall A \geq A_{0} \quad \int_{T_{0}}^{T_{1}} \kappa(A, t) d t \geq \frac{1}{2} \Delta M_{1},
$$

where we have set $\kappa=\kappa_{1}+\kappa_{2}+\kappa_{3}$ and

$$
\begin{gathered}
\kappa_{1}(A, t)=A \int_{A}^{\infty} \int_{A}^{\infty} a\left(y, y^{\prime}\right) f(t, y) f\left(t, y^{\prime}\right) d y d y^{\prime} \geq 0, \\
\kappa_{2}(A, t)=2 \int_{A}^{\infty}\left\{\int_{0}^{A} y a\left(y, y^{\prime}\right) f(t, y) d y\right\} f\left(t, y^{\prime}\right) d y^{\prime} \geq 0,
\end{gathered}
$$




$$
\kappa_{3}(A, t)=\iint_{T_{A}}\left(y+y^{\prime}-A\right) a\left(y, y^{\prime}\right) f(t, y) f\left(t, y^{\prime}\right) d y d y^{\prime} \geq 0,
$$

with $T_{A}$ defined in (3.3).

In order to get estimates (2.14) and (2.15) we treat separately the contribution of each term $\kappa_{i}$. We start showing that the analysis of $\kappa_{3}$ reduces to the analysis of $\kappa_{1}$ and $\kappa_{2}$. Indeed, we have

$$
\begin{aligned}
& \iint_{T_{A}}\left(y+y^{\prime}-A\right) a\left(y, y^{\prime}\right) f(t, y) f\left(t, y^{\prime}\right) d y d y^{\prime}= \\
& =2 \int_{A / 2}^{\infty} d y^{\prime} \int_{0}^{A / 2} d y \mathbf{1}_{T_{A}}\left(y+y^{\prime}-A\right) a\left(y, y^{\prime}\right) f(t, y) f\left(t, y^{\prime}\right) \\
& \quad+\int_{A / 2}^{\infty} \int_{A / 2}^{\infty} \mathbf{1}_{T_{A}}\left(y+y^{\prime}-A\right) a\left(y, y^{\prime}\right) f(t, y) f\left(t, y^{\prime}\right) d y d y^{\prime} \\
& \leq 2 \int_{A / 2}^{\infty}\left\{\int_{0}^{A / 2} y a\left(y, y^{\prime}\right) f(t, y) d y\right\} f\left(t, y^{\prime}\right) d y^{\prime} \\
& \quad+2 \frac{A}{2} \int_{A / 2}^{\infty} \int_{A / 2}^{\infty} a\left(y, y^{\prime}\right) f(t, y) f\left(t, y^{\prime}\right) d y d y^{\prime}
\end{aligned}
$$

since $y+y^{\prime}-A \leq \min \left(y, y^{\prime}, A\right)$ on $T_{A}$. In other words

$$
\kappa_{3}(A, t) \leq \kappa_{2}(A / 2, t)+2 \kappa_{1}(A / 2, t) \quad \forall t, A \geq 0 .
$$

Step 2. We prove (2.14). First notice that for any $\delta_{2}, \tau \geq 0$ with $\delta_{1}>0$ we have

$$
\begin{aligned}
R^{\delta_{1}} \int_{R}^{\infty} f(t, y) y^{\delta_{2}} d y & =\sum_{j \in N} R^{\delta_{1}} \int_{2^{j} R}^{2^{j+1} R} f(t, y) y^{\delta_{2}} d y \\
& \leq \sum_{j \in N}\left(\frac{1}{2^{j\left(\delta_{1}+\tau\right)} R^{\tau}} \int_{2^{j} R}^{2^{j+1} R} f(t, y) y^{\delta_{1}+\delta_{2}+\tau} d y\right) \\
& \leq\left(\sup _{S \geq R} \frac{1}{S^{\tau}} \int_{S}^{2 S} f(t, y) y^{\delta_{1}+\delta_{2}+\tau} d y\right)\left(\sum_{j \in N} \frac{1}{2^{j \delta_{1}}}\right) \\
& \leq C_{\delta_{1}} \sup _{S>R} \frac{1}{S^{\tau}} \int_{0}^{S} f(t, y) y^{\delta_{1}+\delta_{2}+\tau} d y,
\end{aligned}
$$

for some constant $C_{\delta_{1}}$ only depending on $\delta_{1}$.

On the one hand we have for any $\tau \geq 0$

$$
\begin{aligned}
\kappa_{1}(A, t) & =A \int_{A}^{\infty} \int_{A}^{\infty} a\left(y, y^{\prime}\right) f(t, y) f\left(t, y^{\prime}\right) d y d y^{\prime} \\
(5.7) & \leq\left(A^{1 / 2+(\beta-\alpha) / 2} \int_{A}^{\infty} y^{\alpha} f(t, y) d y\right)\left(A^{1 / 2+(\alpha-\beta) / 2} \int_{A}^{\infty} y^{\prime \beta} f\left(t, y^{\prime}\right) d y^{\prime}\right)
\end{aligned}
$$




$$
\leq C_{\alpha, \beta}\left(\sup _{S>A} \frac{1}{S^{\tau}} \int_{0}^{S} f(t, y) y^{\frac{1}{2}+\frac{\lambda}{2}+\tau} d y\right)^{2},
$$

where we have used twice the estimate (5.6), remarking that $|\alpha-\beta|<1$.

On the other hand, for $\left(\alpha^{\prime}, \beta^{\prime}\right)=(\alpha, \beta)$ or $\left(\alpha^{\prime}, \beta^{\prime}\right)=(\beta, \alpha)$ so that $\frac{\alpha^{\prime}-\beta^{\prime}}{2}+\frac{1}{2}>0$, we have, for any $\tau \geq 0$

$$
\begin{aligned}
& \int_{0}^{A} y^{\alpha^{\prime}+1} f(t, y) d y \int_{A}^{\infty} y^{\beta^{\prime}} f\left(t, y^{\prime}\right) d y^{\prime} \leq \\
& \quad \leq\left(\frac{1}{A^{\tau}} \int_{0}^{A} y^{\frac{\lambda}{2}+\frac{1}{2}+\tau} f(t, y) d y\right)\left(A^{\frac{\alpha^{\prime}-\beta^{\prime}}{2}+\frac{1}{2}} \int_{A}^{\infty} y^{\beta^{\prime}} f\left(t, y^{\prime}\right) d y^{\prime}\right) .
\end{aligned}
$$

We then deduce from (5.6) that

$$
\kappa_{2}(A, t) \leq C_{\alpha, \beta}\left(\sup _{S>A} \frac{1}{S^{\tau}} \int_{0}^{S} f(t, y) y^{\frac{1}{2}+\frac{\lambda}{2}+\tau} d y\right)^{2} .
$$

As a conclusion, (2.14) follows from (5.1), (5.5), (5.7) and (5.8) for any $R=$ $A / 2 \geq A_{0} / 2$, and therefore for any $R>0$.

Step 3. We prove (2.15). The lower bound (5.1) and Fubini's theorem (notice that $\kappa_{i} \geq 0$ ) imply

$$
\sum_{i=1}^{3} \int_{T_{0}}^{T_{1}} \int_{e}^{\infty} \frac{\kappa_{i}(A, t)}{A \ln A} d A d t=\int_{e}^{\infty} \int_{T_{0}}^{T_{1}} \kappa(A, t) d t \frac{d A}{A \ln A}=\infty .
$$

From (5.6) we then obtain

$$
\int_{T_{0}}^{T_{1}} \int_{e}^{\infty} \frac{\kappa_{i}(A, t)}{A \ln A} d A d t=+\infty \quad \text { for } i=1 \text { or } 2 .
$$

We need the following lemma, which we state below and prove at the end of the proof of Theorem 2.6.

Lemma 5.1 There is $\xi_{1} \in L^{\infty}\left(\mathbb{R}_{+}^{2}\right)$ such that

$(5.10) \forall y, y^{\prime} \geq e$

$$
\int_{e}^{\min \left(y, y^{\prime}\right)} \frac{d A}{\ln A} \leq 2 \frac{y^{1 / 2+(\beta-\alpha) / 2}}{\sqrt{\ln y}} \frac{y^{\prime 1 / 2-(\beta-\alpha) / 2}}{\sqrt{\ln y^{\prime}}}+\xi_{1}\left(y, y^{\prime}\right) .
$$

For any $\delta \in(0,2)$, there is $\xi_{2} \in L^{\infty}\left(\mathbb{R}_{+}^{2}\right)$ such that

$$
\forall y^{\prime}, z \geq e \quad \int_{e}^{\min \left(y^{\prime}, z\right)} \frac{d A}{A^{1-\delta} \ln A} \leq \frac{2}{\delta} \frac{y^{\prime \delta / 2}}{\sqrt{\ln y^{\prime}}} \frac{z^{\delta / 2}}{\sqrt{\ln z}}+\xi_{2}\left(y^{\prime}, z\right) .
$$

For any $\delta \in(0,2)$, there is $\xi_{3} \in L^{\infty}\left(\mathbb{R}_{+}^{2}\right)$ such that

$$
\forall y, z \geq e \quad \int_{\max (y, z)}^{\infty} \frac{d A}{A^{1+\delta} \ln A} \leq \frac{2}{\delta} \sqrt{\frac{1}{y^{\delta} \ln y}} \sqrt{\frac{1}{z^{\delta} \ln y^{\prime}}}+\xi_{3}(y, z) .
$$


Therefore, using first (5.10) we deduce

$$
\begin{aligned}
\int_{e}^{\infty} \frac{\kappa_{1}(t, A)}{A \ln A} d A & =\int_{e}^{\infty}\left(\int_{A}^{\infty} y^{\alpha} f(t, y) d y \int_{A}^{\infty} y^{\prime \beta} f\left(t, y^{\prime}\right) d y^{\prime}\right) \frac{d A}{\ln A} \\
& =\int_{e}^{\infty} \int_{e}^{\infty} y^{\alpha} f(t, y) y^{\prime \beta} f\left(t, y^{\prime}\right)\left(\int_{e}^{\min \left(y, y^{\prime}\right)} \frac{d A}{\ln A}\right) d y d y^{\prime} \\
& \leq\left(\int_{e}^{\infty} \frac{y^{\lambda / 2+1 / 2}}{(\ln y)^{1 / 2}} f(t, y) d y\right)^{2}+\|\xi\|_{L^{\infty}}\|f(t, .)\|_{L_{1}^{1}}^{2} .
\end{aligned}
$$

On the other hand, using Cauchy-Schwarz inequality, we have

$$
\begin{aligned}
& \int_{e}^{\infty} \frac{d A}{A \ln A}\left(\int_{0}^{A} y^{\alpha^{\prime}+1} f(t, y) d y\right)\left(\int_{A}^{\infty} y^{\prime \beta^{\prime}} f\left(t, y^{\prime}\right) d y^{\prime}\right) \leq \\
& \leq\left[\int_{e}^{\infty} \frac{1}{A^{1+\delta} \ln A}\left(\int_{0}^{A} y^{\alpha^{\prime}+1} f(t, y) d y\right)^{2} d A\right]^{1 / 2} \\
& \quad\left[\int_{e}^{\infty} \frac{1}{A^{1-\delta} \ln A}\left(\int_{A}^{\infty} y^{\prime \beta^{\prime}} f\left(t, y^{\prime}\right) d y^{\prime}\right)^{2} d A\right]^{1 / 2}
\end{aligned}
$$

with $\delta=1-(\beta-\alpha)$ if $\left(\alpha^{\prime}, \beta^{\prime}\right)=(\alpha, \beta)$ and $\delta=1+\beta-\alpha$ if $\left(\alpha^{\prime}, \beta^{\prime}\right)=(\beta, \alpha)$.

Notice that in both cases $\delta \in(0,2]$.

First, using (5.12), it yields

$$
\begin{aligned}
K_{1}(t) & :=\int_{e}^{\infty} \frac{1}{A^{1+\delta} \ln A}\left(\int_{0}^{A} y^{\alpha^{\prime}+1} f(t, y) d y\right)^{2} d A \\
& \leq 2 \int_{e}^{\infty} \int_{e}^{\infty} y^{\alpha^{\prime}+1} f(t, y) z^{\alpha^{\prime}+1} f(t, z) \int_{\max (y, z)}^{\infty} \frac{d A}{A^{1+\delta} \ln A} d y d z \\
& +2 \int_{e}^{\infty} \frac{1}{A^{1+\delta} \ln A}\left(\int_{0}^{e} y^{\alpha^{\prime}+1} f(t, y) d y\right)^{2} d A \\
& \leq \frac{2}{\delta}\left(\int_{e}^{\infty} \frac{y^{\lambda / 2+1 / 2}}{(\ln y)^{1 / 2}} f(t, y) d y\right)^{2}+\left(\left\|\xi_{3}\right\|_{L^{\infty}}+C_{\delta}\right)\|f(t, .)\|_{L_{1}^{1}}^{2}
\end{aligned}
$$

for some positive constant $C_{\delta}$ only depending on $\delta$.

Next, using (5.11), we get

$$
\begin{aligned}
K_{2}(t) & :=\int_{e}^{\infty} \frac{1}{A^{1-\delta} \ln A}\left(\int_{A}^{\infty} y^{\beta^{\prime}} f\left(t, y^{\prime}\right) d y^{\prime}\right)^{2} d A \\
& =\int_{e}^{\infty} \int_{e}^{\infty} y^{\prime \beta^{\prime}} f\left(t, y^{\prime}\right) z^{\beta^{\prime}} f(t, z) \int_{e}^{\min \left(y^{\prime}, z\right)} \frac{d A}{A^{1-\delta} \ln A} d y^{\prime} d z \\
& \leq \frac{2}{\delta}\left(\int_{e}^{\infty} \frac{y^{\lambda / 2+1 / 2}}{(\ln y)^{1 / 2}} f(t, y) d y\right)^{2}+\left\|\xi_{2}\right\|_{L^{\infty}}\|f(t, .)\|_{L_{1}^{1}}^{2} .
\end{aligned}
$$

Gathering (5.14), (5.15), (5.16) and using Young's inequality we deduce

$$
\int_{T_{0}}^{T_{1}} \int_{e}^{\infty} \frac{\kappa_{2}(t, A)}{A \ln A} d A d t \leq \frac{1}{2} \int_{T_{0}}^{T_{1}} K_{1}(t) d t+\frac{1}{2} \int_{T_{0}}^{T_{1}} K_{2}(t) d t
$$




$$
\leq \frac{2}{\delta} \int_{T_{0}}^{T_{1}}\left(\int_{e}^{\infty} \frac{y^{\lambda / 2+1 / 2}}{(\ln y)^{1 / 2}} f(t, y) d y\right)^{2} d t+T_{1} C_{\xi_{2}, \xi_{3}}\|f(0, .)\|_{L_{1}^{1}}^{2} .
$$

As a conclusion, (2.15) follows from (5.9), (5.13), (5.17).

Proof of Lemma 5.1. We only prove (5.10) since (5.11) and (5.12) follow in the same way. Integrating by part, we have

$$
\int_{e}^{\min \left(y, y^{\prime}\right)} \frac{d A}{\ln A}=\frac{\min \left(y, y^{\prime}\right)}{\ln \left(\min \left(y, y^{\prime}\right)\right)}-1+\int_{e}^{\min \left(y, y^{\prime}\right)} \frac{d A}{(\ln A)^{2}} .
$$

Since for any $k \in(0,2)$ there is $A_{k} \geq 0$ such that $z \mapsto \frac{z^{k}}{\ln z}$ is increasing for $z \geq A_{k}$, we also have

$$
\begin{aligned}
\frac{\min \left(y, y^{\prime}\right)}{\ln \left(\min \left(y, y^{\prime}\right)\right)} & =\min \left(\frac{y}{\ln y}, \frac{y^{\prime}}{\ln y^{\prime}}\right) \leq y^{\beta-\alpha} \min \left(\frac{y^{1-(\beta-\alpha)}}{\ln y}, \frac{y^{\prime 1-(\beta-\alpha)}}{\ln y^{\prime}}\right) \\
& \leq \frac{y^{1 / 2+(\beta-\alpha) / 2}}{\sqrt{\ln y}} \frac{y^{1 / 2-(\beta-\alpha) / 2}}{\sqrt{\ln y^{\prime}}}
\end{aligned}
$$

for any $y, y^{\prime} \geq \max \left(A_{0}, A_{1-(\beta-\alpha)}\right)$. We conclude observing that the last term in the right hand side of (5.18) is bounded by one half of the left hand side term of (5.18) for large value of $\min \left(y, y^{\prime}\right)$.

Remark 5.2 Let emphasize that for any $g \geq 0$ none of the two informations on the behavior of $g(y)$ for large value $y \geq 0$

$$
M C_{\tau}(g):=\limsup _{R \rightarrow \infty} \frac{1}{R^{\tau}} \int_{0}^{R} y^{\tau} g(y) d y>0
$$

and

$$
L_{\delta}(g):=\int_{e}^{\infty} \frac{g(y)}{(\ln y)^{\delta}} d y=+\infty
$$

is stronger than the other, as it is shown by the two examples below. In particular, (2.14) and (2.15) can not be deduced one from the other. On the one hand, taking

$$
g(y)=\sum_{j \in \mathbb{N}^{*}} \lambda_{j} \delta_{y_{j}}, \quad \lambda_{j}=1, \quad y_{j}=e^{j^{\omega}}
$$

we have

$$
M C_{\tau}(g)=\sum_{k \in \mathbb{N}^{*}} e^{-\tau k^{\omega}}>0 \quad \text { and } \quad L_{\delta}(g)=\sum_{j \in \mathbb{N}^{*}} j^{-\omega \delta}<\infty \text { if } \omega \delta>1 .
$$

On the other hand, choosing $g(y)=y^{-1}(\ln y)^{-\nu}$, we get

$$
M C_{\tau}(g)=0 \quad \text { and } \quad L_{\delta}(g)=+\infty \text { if } \nu+\delta \leq 1 .
$$


Proof of Corollary 1.3. From (1.22) we obtain by Fatou's lemma

$$
\int_{T_{0}}^{T_{1}}\left(\liminf _{R \rightarrow \infty} \frac{1}{R^{\tau}} \int_{0}^{R} f(t, y) y^{\lambda / 2+1 / 2+\tau} d y\right)^{2} d t \leq C_{1} .
$$

Then if we define the measurable function: $\omega_{\tau}: \mathbb{R}_{+} \rightarrow \mathbb{R}_{+}$by

$$
\omega_{\tau}(t):=\tau \liminf _{R \rightarrow \infty} \frac{1}{R^{\tau}} \int_{0}^{R} f(t, y) y^{\lambda / 2+1 / 2} d y \quad \text { for a.e. } \quad t \in\left[T_{0}, T_{1}\right]
$$

we deduce

$$
\liminf _{y \rightarrow \infty} f(t, y) y^{\lambda / 2+3 / 2} \leq \omega_{\tau}(t) \quad \text { for a.e. } \quad t \in\left[T_{0}, T_{1}\right] .
$$

Gathering (5.19) with (1.24) we get the second estimate in (1.25).

On the other hand, we deduce from (1.23) and (1.24):

$$
\left(T_{1}-T_{0}\right) \Lambda^{2}\left(\limsup _{R \rightarrow \infty} \frac{1}{R^{\tau}} \int_{0}^{R} \xi(y) y^{\lambda / 2+1 / 2+\tau} d y\right)^{2} \geq C_{1}^{-1},
$$

which implies

$$
\limsup _{y \rightarrow \infty} \xi(y) y^{\lambda / 2+3 / 2} \geq\left(\frac{1}{\tau C_{1}^{1 / 2} \Lambda\left(T_{1}-T_{0}\right)^{1 / 2}}\right),
$$

and this ends the proof of Corollary 1.3.

\section{Behavior of solutions to the coagulation frag- mentation equation}

This section is devoted to the proof of Theorem 2.7, Theorem 1.4 and Corollary 2.9 .

Proof of Theorem 2.7. Consider a weak solution $f$ to the coagulation and fragmentation equation (1.27). For any $\psi \in L^{\infty}\left(\mathbb{R}_{+}\right)$and $t_{1} \geq t_{0} \geq 0$ we get from the fundamental identities (1.5) and (1.32)

$$
\begin{array}{r}
\int_{0}^{\infty} f\left(t_{1}, y^{\prime}\right) \psi\left(y^{\prime}\right) d y^{\prime}+\frac{1}{2} \int_{t_{0}}^{t_{1}} \int_{0}^{\infty} \int_{0}^{\infty} a f(t, y) f\left(t, y^{\prime}\right) \tilde{\psi} d y d y^{\prime} d t= \\
=\int_{0}^{\infty} f\left(t_{0}, y^{\prime}\right) \psi\left(y^{\prime}\right) d y^{\prime}+\frac{1}{2} \int_{t_{0}}^{t_{1}} \int_{0}^{\infty} f(t, z) B(z) K_{\psi}(z) d z
\end{array}
$$

with

$$
K_{\psi}(z)=\int_{0}^{z}(\psi(y)+\psi(z-y)-\psi(z)) d y .
$$


We proceed in two steps.

Step 1. Choose $\psi(y)=\psi_{A}(y)=y \wedge A$, so that $\tilde{\psi}=\tilde{\psi}_{A}$ given by (3.2) and $K_{A}(z)=A(z-A)^{+}$. We obtain, arguing as for the proof of 3.4

$$
\frac{1}{2} \int_{t_{0}}^{t_{1}}\left(\int_{A}^{\infty} f(t, y) y^{\lambda / 2} d y\right)^{2} d t \leq \frac{M_{1}\left(t_{0}\right)}{A}+\frac{B}{2} \int_{t_{0}}^{t_{1}} \int_{A}^{\infty} f(t, z) \frac{z}{(1+z)^{\gamma}} d z d t .
$$

For the last term, using condition (1.31) and the Young inequality, we get

$$
\begin{gathered}
\int_{A}^{\infty} f(t, z) z^{\lambda / 2}\left\{\frac{1}{z^{1 / 2}} \frac{z^{3 / 2-\lambda / 2}}{(1+z)^{\gamma}}\right\} d z \leq \frac{1}{A^{1 / 2}} \int_{A}^{\infty} f(t, z) z^{\lambda / 2} d z \\
\leq \frac{1}{2 B}\left(\int_{A}^{\infty} f(t, z) z^{\lambda / 2} d z\right)^{2}+\frac{B}{2 A} .
\end{gathered}
$$

Gathering the two preceding estimates we deduce that $\forall A>0$

$$
\int_{t_{0}}^{t_{1}}\left(\int_{A}^{\infty} f(t, y) y^{\lambda / 2} d y\right)^{2} d t \leq \frac{1}{A}\left[4 M_{1}\left(t_{0}\right)+B^{2}\left(t_{1}-t_{0}\right)\right] .
$$

Proceeding as in the proof of Theorem 2.2 and Corollary 2.3 we readily deduce (2.18) and then (2.19), (2.20).

Step 2. In order to prove (2.21) we make the choice $\psi=1$ in (6.1), so that $\tilde{\psi}=1, K_{\psi}(z)=z$, and thanks to $(1.15)$, we get

$$
\frac{1}{2} \int_{t_{0}}^{t_{1}} M_{\lambda / 2}^{2}(t) d t \leq M_{0}\left(t_{0}\right)+\frac{B}{2} \int_{t_{0}}^{t_{1}} \int_{0}^{\infty} f(t, z) \frac{z}{(1+z)^{\gamma}} d z d t .
$$

Using once again condition (1.31) and the Young inequality we have

$$
\int_{0}^{\infty} f(t, z) \frac{z}{(1+z)^{\gamma}} d z \leq \frac{1}{2 B} M_{\lambda / 2}^{2}(t)+\frac{B}{2},
$$

from where we obtain

$$
\int_{t_{0}}^{t_{1}} M_{\lambda / 2}^{2}(t) d t \leq 4 M_{0}\left(t_{0}\right)+B^{2}\left(t_{1}-t_{0}\right) .
$$

Estimate (2.21) just follows interpolating (6.4) and (2.20).

Proof of Theorem 1.4. We proceed in two steps.

Step 1: proof of (1.36) By (2.21) with $k=1$ we have

$$
\int_{0}^{T} M_{1}^{2}(t) d t \leq C_{1}\left(M_{0}(0)+M_{1}(0)+B^{2} T\right) .
$$

This inequality may holds with $M_{1}(t) \equiv M_{1}(0)$ for any time $T \geq 0$ only if $M_{1}(0) \leq \sqrt{C_{1}} B$. When $M_{1}(0)>\sqrt{C_{1}} B$, gelation must occurs before the time

$$
T_{*}=\frac{C_{1}\left(M_{0}(0)+M_{1}(0)\right)}{M_{1}^{2}(0)-C_{1} B^{2}} .
$$


Step 2: We prove (1.38). Coming back to formula (6.1) with $\psi(y)=y \wedge A$ we have

$$
\begin{gathered}
\int_{0}^{\infty} f\left(t_{1}, y\right) y \wedge A d y+\frac{1}{2} \int_{t_{0}}^{t_{1}} \int_{0}^{\infty} \int_{0}^{\infty} a f(t, y) f\left(t, y^{\prime}\right)\left(-\tilde{\psi}_{A}\right) d y d y^{\prime} d t \leq \\
\leq \int_{0}^{\infty} f\left(t_{0}, y\right) y \wedge A d y+\frac{1}{2} \int_{t_{0}}^{t_{1}} \int_{0}^{\infty} f(t, z) B(z) K_{A}(z) d z d t
\end{gathered}
$$

with $\tilde{\psi}=\tilde{\psi}_{A}$ given by $(3.2)$ and $K_{A}(z)=A(z-A)^{+}$. We assume by contradiction that $M_{1}(t) \equiv M_{1}(0)$. Therefore, for any $A>0$,

$$
\int_{0}^{A} y f(t, y) d y \geq \frac{M_{1}(0)}{2} \text { or } \int_{A}^{\infty} y f(t, y) d y \geq \frac{M_{1}(0)}{2} .
$$

We deduce from (6.6) that for $A$ large enough

$$
\begin{aligned}
& \int_{0}^{\infty} f(t, z) B(z) K_{A}(z) d z \leq B \int_{A}^{\infty} f(t, z) z^{1-\gamma} A d z \\
& \leq \min \left\{\frac{B}{A^{\gamma-\alpha}} \int_{A}^{\infty} A r\left(y^{\prime}\right)^{\alpha} f\left(t, y^{\prime}\right) d y^{\prime}, \frac{B}{A^{\gamma-1}} \int_{A}^{\infty} r(y) f(t, y) d y\right\} \\
& \leq \frac{M_{1}(0)}{4} \min \left(\int_{A}^{\infty} A r\left(y^{\prime}\right)^{\alpha} f\left(t, y^{\prime}\right) d y^{\prime}, \int_{A}^{\infty} r(y) f(t, y) d y\right) \\
& \leq \frac{1}{2}\left(A \int_{A}^{\infty} r(y) f(t, y) d y \int_{A}^{\infty} r\left(y^{\prime}\right)^{\alpha} f\left(t, y^{\prime}\right) d y^{\prime} d t\right. \\
& \left.\quad+\int_{0}^{A} y r(y)^{\alpha} f(t, y) d y \int_{A}^{\infty} r\left(y^{\prime}\right) f\left(t, y^{\prime}\right) d y^{\prime}\right) \\
& \leq \frac{1}{2} \int_{0}^{\infty} \int_{0}^{\infty} a f(t, y) f\left(t, y^{\prime}\right)\left(-\tilde{\psi}_{A}\left(y, y^{\prime}\right)\right) d y d y^{\prime} .
\end{aligned}
$$

This imply that there exists $A_{0}$ large enough such that for any $A \geq A_{0} / 2$ and any $t_{1} \geq t_{1} \geq 0$

$$
\frac{1}{4} \int_{t_{0}}^{t_{1}} \int_{0}^{\infty} \int_{0}^{\infty} a f(t, y) f\left(t, y^{\prime}\right)\left(-\tilde{\psi}_{A}\right) d y d y^{\prime} d t \leq M_{1}\left(t_{0}\right)
$$

and

$$
\int_{0}^{\infty} f\left(t_{1}, y\right) y \wedge A d y \leq \int_{0}^{\infty} f\left(t_{0}, y\right) y \wedge A d y .
$$

From (6.7) we deduce (as in the proof of 2.5) that

$$
t \mapsto \int_{A_{0}}^{\infty} f(t, y) y d y \in L^{2}(0, \infty)
$$


and from (6.8) we deduce that for any $t \geq 1$

$$
\begin{aligned}
\int_{A_{0}}^{\infty} f(t, y) y d y & \geq \int_{0}^{\infty}\left(y-A_{0}\right)^{+} f(t, y) d y \\
& \geq M_{1}(0)-\int_{0}^{\infty}\left(y \wedge A_{0}\right) f(t, y) d y \\
& \geq M_{1}(0)-\int_{0}^{\infty}\left(y \wedge A_{0}\right) f(1, y) d y
\end{aligned}
$$

From Lemma 6.1 whose statement and proof are given just below, we know that $f(1, y)>0$ a.e. on $\mathbb{R}_{+}$and the right hand side in (6.10) is a positive constant. We deduce that (6.9) and (6.10) can not hold together and we have a contradiction.

Step 3: We prove (1.37). We easily deduce of assumption (1.38) that for $A_{0}$ large enough, so that supp $B \subset\left[0, A_{0}\right],(6.7)$ and (6.8) still hold. We then conclude as at the end of the preceding step.

Lemma 6.1 Every weak solution of the coagulation-fragmentation equation (1.27) with kinetic kernels such that

$$
0<a\left(y, y^{\prime}\right), b\left(y, y^{\prime}\right) \leq C(1+y)\left(1+y^{\prime}\right) \text { for a.e. } y, y^{\prime} \in \mathbb{R}_{+}
$$

and not identically zero initial data satisfies

$$
f(t, .)>0 \text { a.e. on } \mathbb{R}_{+} \text {for any } t>0 .
$$

Proof of Lemma 6.1. With the assumptions made on the kinetic kernels we have

$$
\frac{\partial f}{\partial t}+\lambda(t, y) f(t, y) \geq \Sigma(t, y) \text { on } \mathbb{R}_{+}^{2},
$$

with

$$
\begin{aligned}
& \text { (6.14) } \lambda(t, y):=\frac{1}{2} \int_{0}^{y} b\left(y-y^{\prime}, y^{\prime}\right) d y^{\prime}+C(1+y)\|f(t, .)\|_{L_{1}^{1}} \in L_{l o c}^{\infty}\left(\mathbb{R}_{+}^{2}\right) \\
& (6.15) \Sigma(t, y):=\frac{1}{2} \int_{0}^{y} a\left(y-y^{\prime}, y^{\prime}\right) f\left(t, y-y^{\prime}\right) f\left(t, y^{\prime}\right) d y^{\prime} \\
& +\int_{0}^{\infty} b\left(y, y^{\prime}\right) f\left(t, y+y^{\prime}\right) d y^{\prime} \in L_{l o c}^{1}\left(\mathbb{R}_{+}^{2}\right) .
\end{aligned}
$$

We proceed in several steps.

Step 1. First, by hypothesis there exists $a>0$ such that $f_{i n} \not \equiv 0$ a.e. on $(2 a, 3 a)$. Therefore, since $\Sigma \geq 0$, equation (6.13) implies that $f(t,.) \not \equiv 0$ a.e. on $(2 a, 3 a)$ and for any $t \geq 0$. 
Step 2. Thanks to step 1, we have for a.e. $y \in(0,2 a)$ and any $t \geq 0$

$$
\Sigma(t, y) \geq \int_{0}^{\infty} b\left(y, y^{\prime}\right) f\left(t, y+y^{\prime}\right) d y^{\prime} \geq \int_{2 a}^{3 a} b(y, z-y) f(t, z) d z>0,
$$

and then equation (6.13) implies that $f(t,)>$.0 a.e. on $(0,2 a)$ for any $t>0$. Step 3. Now, thanks to step 2, we have for a.e. $y \in(0,4 a)$ and any $t>0$

$$
\Sigma(t, y) \geq \frac{1}{2} \int_{0}^{y} a\left(y-y^{\prime}, y^{\prime}\right) f\left(t, y-y^{\prime}\right) f\left(t, y^{\prime}\right) d y^{\prime}>0,
$$

and therefore equation(6.13) implies that $f(t,)>$.0 a.e. on $(0,4 a)$ for any $t>0$.

Step 4. Assertion (6.12) follows iterating the step 3.

Proof of Corollary 2.9. Let $f$ be a solution to the coagulation-fragmentation equation (1.27). Then it satisfies (2.20). Coming back to (6.5), we notice that for $\varepsilon \in(0, \gamma+\lambda / 2-3 / 2)$ we have

$$
\begin{aligned}
\int_{0}^{\infty} f(t, z) B(z) K_{A}(z) d z & \leq \int_{A}^{\infty} f(t, z) z^{2-\gamma} d z \\
& \leq \frac{1}{A^{\gamma+\lambda / 2-3 / 2-\varepsilon}} M_{\frac{\lambda}{2}+\frac{1}{2}-\varepsilon}(t) \underset{A \rightarrow \infty}{\longrightarrow} 0
\end{aligned}
$$

in $L^{1}(0, T)$ for any $T \in \mathbb{R}_{+}$. Letting $A \rightarrow \infty$ in (6.5) we first deduce from (6.16) that $M_{1}(t)$ is decreasing. Moreover, since (4.10) still holds (because the contribution of the fragmentation term has the good sign) we deduce that $M_{1}(t)$ is right continuous. Finally, if $\Delta M_{1}:=M_{1}\left(t_{0}\right)-M_{1}\left(t_{1}\right)>0$ we deduce from (6.5) and (6.16) that

$$
\int_{t_{0}}^{t_{1}} \int_{0}^{\infty} \int_{0}^{\infty} a f(t, y) f\left(t, y^{\prime}\right)\left(-\tilde{\psi}_{A}\right) d y d y^{\prime} d t \geq \frac{\Delta M_{1}}{2}
$$

for $A$ large enough. Therefore, the analysis performed in the proof of Theorem 2.6 still holds, so that (2.14) and (2.15) follow.

\section{$7 \quad$ Existence result}

This section is devoted to the proof of Corollary 2.8. Like in [23] and [26], the strategy is to define a sequence $\left(f_{n}\right)$ of solutions to the coagulation-fragmentation equation with "truncated" coefficients, to establish some bounds which hold uniformly in $n \geq 0$ and then pass to the limit in a weak formulation of solutions to the equations.

Let us define the approximated coagulation kernel

$$
a_{n}\left(y, y^{\prime}\right):=a\left(y \wedge n, y^{\prime} \wedge n\right) \text { and } b_{n}\left(y, y^{\prime}\right):=b\left(y, y^{\prime}\right) \mathbf{1}_{y+y^{\prime} \leq n}
$$


and denote by $f_{n} \in C\left([0, \infty) ; L^{1}\right) \cap L^{\infty}\left(0, T ; L_{1}^{1}\right)$ for any $T>0$ the weak solution to the coagulation-fragmentation equation (1.27) associated to (7.1), $b$ and $f_{i n}$. Such a solution exists thanks to a standard Banach fix point theorem, see for instance [31].

Since $a_{n}\left(y, y^{\prime}\right), b_{n}\left(y, y^{\prime}\right) \leq C(1+y)\left(1+y^{\prime}\right)$ uniformly in $n$, it has been proved in [26] that the sequence $\left(f_{n}\right)$ satisfies the following estimates

$$
M_{1}\left(f_{n}(t, .)\right) \leq M_{1}\left(f_{\text {in }}\right), \quad M_{0}\left(f_{n}(t, .)\right) \leq C_{T} \quad \forall t \in[0, T],
$$

and for any $R, T>0$ there exits a function $\Psi_{R}$ such that $\Psi_{R}(s) / s \rightarrow \infty$ when $s \rightarrow \infty$ and

$$
\sup _{n} \sup _{t \in[0, T]} \int_{0}^{R} \Psi_{R}\left(f_{n}(t, y)\right) d y \leq C_{T, R}<\infty .
$$

We need one additional moment estimate that we derive now. Let us fix $T \geq 0$. Proceeding as at the beginning of Step 1 in the proof of Theorem (2.7) we get

$$
\begin{aligned}
& \frac{1}{2} \int_{0}^{T}\left(\int_{A}^{\infty} f(t, y)(y \wedge n)^{\lambda / 2} d y\right)^{2} d t \leq \\
& \quad \leq M_{1}(0)+\frac{1}{2} \int_{0}^{T} \int_{A}^{\infty} f_{n}(t, z) \frac{(y \wedge n)^{\lambda / 2}}{A^{1 / 2}} d z d t,
\end{aligned}
$$

for any $A>0$. Then (using Young inequality) we may follow the proof of (2.20) in Theorem 2.7 to obtain

$$
\sup _{n \geq 0} \int_{0}^{T}\left(\int_{e}^{\infty} f_{n}(t, y)(y \wedge n)^{\lambda / 2} \frac{y^{1 / 2}}{(\ln y)^{2}} d y\right)^{2} d t \leq C_{T} .
$$

By (7.2) and (7.3) it is straightforward that $\left(f_{n}\right)$ lies in a weak compact set of $L^{1}\left((0, T) \times \mathbb{R}_{+}\right.$for any $T>0$. Therefore, there exists a function $f \in$ $C\left([0, \infty) ; L^{1}\right) \cap L^{\infty}\left(0, T ; L_{1}^{1}\right)$ for all $T>0$ such that for a subsequence of $\left(f_{n}\right)$ (not relabeled) $f_{n} \rightarrow f$ weakly in $L^{1}\left((0, T) \times \mathbb{R}_{+}\right)$for any $T>0$. Moreover, it is possible to show that the coagulation and fragmentation kernels $Q_{f, n}\left(f_{n}\right)$ and $Q_{c, n}\left(f_{n}\right)$ lie in a weak compact set of $L^{1}((0, T) \times(0, R)$ for any $T, R>0$ and for any $T, R>0$ :

$$
Q_{f, n}\left(f_{n}\right), Q_{c, n}\left(f_{n}\right) \rightarrow Q_{f}(f), Q_{c}(f) \text { weakly in } L^{1}((0, T) \times(0, R)) .
$$

Since the fragmentation term is treated for instance in [23], we only briefly explain how to deal with the coagulation term. We refer to [26] for more details.

Let fix $\psi \in \mathcal{D}\left([0, \infty) \times \mathbb{R}_{+}\right), M>0$ such that $\operatorname{supp} \psi \subset[0, \infty) \times[0, M]$ and $R \geq M$. Using (1.5) we have

$$
\int_{0}^{\infty} Q_{c, n}\left(f_{n}(t, .)\right) \psi d y=\frac{1}{2} \int_{0}^{\infty} \int_{0}^{\infty} a_{n}\left(y, y^{\prime}\right) f_{n}(t, y) f\left(t, y^{\prime}\right) \tilde{\psi}\left(t, y, y^{\prime}\right) d y d y^{\prime}
$$




$$
\begin{aligned}
& =\frac{1}{2} \int_{0}^{R} \int_{0}^{R} a_{n}\left(y, y^{\prime}\right) f_{n}(t, y) f\left(t, y^{\prime}\right) \tilde{\psi}\left(t, y, y^{\prime}\right) d y d y^{\prime} \\
& +\frac{1}{2} \iint_{\mathbb{R}_{+}^{2} \backslash[0, R]^{2}} a_{n} f_{n}(t, y) f\left(t, y^{\prime}\right)\left(\psi(t, y)+\psi\left(t, y^{\prime}\right)\right) d y d y^{\prime} .
\end{aligned}
$$

On the one hand, using Lemma 3.5 and Lemma 4.4 in [26], we can pass to the limit in the first term, so that

$$
\begin{aligned}
\int_{0}^{T} \int_{0}^{R} \int_{0}^{R} a_{n}\left(y, y^{\prime}\right) f_{n}(t, y) f_{n}\left(t, y^{\prime}\right) \tilde{\psi}\left(t, y, y^{\prime}\right) d y d y^{\prime} d t \\
\quad \underset{n \rightarrow \infty}{\longrightarrow} \int_{0}^{T} \int_{0}^{R} \int_{0}^{R} a\left(y, y^{\prime}\right) f(t, y) f\left(t, y^{\prime}\right) \tilde{\psi}\left(t, y, y^{\prime}\right) d y d y^{\prime} d t .
\end{aligned}
$$

On the other hand, using Cauchy-Schwarz inequality, we have

$$
\begin{aligned}
& \int_{0}^{T} \iint_{\mathbb{R}_{+}^{2} \backslash[0, R]^{2}} a_{n} f_{n}(t, y) f_{n}\left(t, y^{\prime}\right)\left(\psi(y)+\psi\left(y^{\prime}\right)\right) d y d y^{\prime} d t \leq \\
& \leq C_{a}\|\psi\|_{\infty} \int_{0}^{T} \int_{0}^{M}(1+y) f_{n} d y \int_{R}^{\infty}(y \wedge n)^{\beta} f_{n} d y^{\prime} d t \\
& \leq C_{a, \psi} \sup _{[0, T]}\left\|f_{n}(t, .)\right\|_{L_{1}^{1}} \sqrt{T}\left(\int_{0}^{T}\left(\int_{R}^{\infty}(y \wedge n)^{\beta} f_{n} d y^{\prime}\right)^{2} d t\right)^{1 / 2} \\
& \leq \frac{C_{a, \psi, f_{i n}, T}}{R^{\frac{\lambda}{2}+\frac{1}{2}-\beta-\varepsilon}}\left(\int_{0}^{T}\left(\int_{R}^{\infty}(y \wedge n)^{\frac{\lambda}{2}+\frac{1}{2}-\varepsilon} f_{n} d y^{\prime}\right)^{2} d t\right)^{1 / 2} \longrightarrow 0,
\end{aligned}
$$

as $R \rightarrow \infty$, uniformly in $n$. By (7.6) and (7.7) the coagulation term satisfies (7.5). Then with (7.5) at hand, we easily pass to the limit in the weak formulation of equation (1.27) satisfied by $\left(f_{n}\right)$ and we obtain that $f$ is a weak solution in the sense of Definition 2.2.

Remark 7.1 Theorem 2.7 readily extends to a coagulation kernel of the form

$$
\left\{\begin{array}{c}
a=a_{1}+a_{2}, a_{1} \text { satisfying }(1.2), \quad a_{2} \text { is symetric, such that } \\
0 \leq a_{2} \leq C(1+y)^{-1 / 2-\lambda / 2-\varepsilon}\left(1+y^{\prime}\right)^{-1 / 2-\lambda / 2-\varepsilon} \text { for some } \varepsilon>0 .
\end{array}\right.
$$

\section{Extensions to non-homogeneous models}

Almost all the results obtained in the previous sections extend to a non spatially homogeneous setting under suitable conditions. We briefly explain in this section how this can be done. Let us emphasize that the questions of gelation and gelling profile in a non spatially homogeneous setting have been adressed in [19] in the case $\alpha=\beta=1$. 
Now, the clusters are assumed to move in an open bounded subset $\Omega$ of $\mathbb{R}^{N}, N \geq 1$, with smooth boundary $\partial \Omega$, according to brownian movement with diffusion coefficient $d$ only size dependent. We assume $d \in C\left(\mathbb{R}_{+}\right)$and $d(y) \geq$ $0 \forall y>0$. We denote by $f(t, x, y) \geq 0$ the distribution of clusters of size $y \in \mathbb{R}_{+}$ at time $t \geq 0$ and position $x \in \Omega$. The continuous coagulation-fragmentation equation with diffusion reads

$$
\left\{\begin{array}{cl}
\frac{\partial f}{\partial t}-d(y) \Delta_{x} f & =Q_{c}(f)+Q_{f}(f), \quad \text { in }(0,+\infty) \times \Omega \times \mathbb{R}_{+} \\
\frac{\partial f}{\partial n} & =0, \quad \text { on }(0,+\infty) \times \partial \Omega \times \mathbb{R}_{+} \\
f(0, x, y) & =f_{\text {in }}(x, y), \quad \text { in } \Omega \times \mathbb{R}_{+}
\end{array}\right.
$$

Here, $\partial_{n} f$ denotes the outward normal derivative of $f$ on the boundary and the terms $Q_{c}(f)$ and $Q_{f}(f)$ are given in (1.1) and (1.28).

Under the assumptions (1.2)-(1.3) on $a$, (1.29)-(1.30) with $\gamma>1$ on $b$ and $f_{\text {in }} \in L^{1}\left(\Omega \times \mathbb{R}_{+},(1+y) d y d x\right)$, it has been proved in [25] that there exists a weak global solution in the following sense:

$$
\begin{aligned}
& f \in C\left([0, T) ; L^{1}\left(\Omega \times \mathbb{R}_{+}\right) \cap L^{\infty}\left(0, T ; L^{1}\left(\Omega \times \mathbb{R}_{+} ; y d y d x\right)\right),\right. \\
& f \in \cap L^{1}\left((0, T) \times(0, R) ; W^{1,1}(\Omega)\right), \\
& Q(f) \in L^{1}\left((0, T) \times \Omega \times \mathbb{R}_{+}\right)
\end{aligned}
$$

for all $T>0$, and satisfies the following weak formulation of (8.1)

$$
\begin{aligned}
& \int_{\Omega} \int_{0}^{\infty}\left(\psi(t) f(t)-\psi(0) f_{\text {in }}\right) d y d x \\
& \quad+\int_{0}^{t} \int \Omega \int_{0}^{\infty}\left(d(y) \nabla_{x} f \nabla_{x} \psi-f \partial_{t} \psi\right) d y d x d s \\
& =\frac{1}{2} \int_{0}^{t} \int_{\Omega} \int_{0}^{\infty} Q(f) \psi d y d x d s,
\end{aligned}
$$

for every $t \in(0, T)$ and all $\psi \in W^{1, \infty}\left([0, T] \times \bar{\Omega} \times \mathbb{R}_{+}\right)$with compact support in $[0, T] \times \bar{\Omega} \times \mathbb{R}_{+}$. The case $\alpha \in(0,1), \beta=1$ is not actually considered in [25], but the existence result in [25] can be extended to this case adapting Corollary 2.8. We refer to [25] for details about the definition of solutions as well as for the precise statement of the existence result. We also refer to [24] and references therein for the existence of solution to the discrete coagulation-fragmentation equations with diffusion.

We finally introduce the following notation

$$
\mathcal{M}_{k}(t):=\int_{\Omega} M_{k}(t, x) d x, \quad M_{k}(t, x):=M_{k}(f(t, x, .)) .
$$

In that context, the gelation time is now defined as the smallest time $T_{g}$ satisfying that, for every $t_{0} \geq 0, t_{1} \geq 0$ such that $t_{0}<T_{g}<t_{1}$ there holds $\mathcal{M}_{1}\left(t_{1}\right)<\mathcal{M}_{1}\left(t_{0}\right)$. 
We now state the extension of some of the results obtained in the previous Sections (Theorem 2.2, Corollary 2.3, Theorem 2.4 in the pure coagulation case, Theorem 2.7 and part of Theorem 1.4 in the coagulation-fragmentation case) to this non homogeneous setting. Since their proofs are rather straightforward extensions of those for the homogeneous equations, we skip them for the sake of brevity.

Theorem 8.1 Let $\Phi: \mathbb{R}_{+} \rightarrow \mathbb{R}_{+}$be an increasing function satisfying $\Phi(0)=0$ and (2.3). For any weak solution $f$ to (8.1) and any $t_{1} \geq t_{0} \geq 0$ the following non homogeneous version of (2.18) holds

$$
\begin{array}{r}
\int_{t_{0}}^{t_{1}} \int_{\Omega}\left(\int_{0}^{\infty} f(t, x, y) y^{\lambda / 2} \Phi(y) d y\right)^{2} d x d t \leq \\
\leq C_{\Phi}^{2}\left[4 \mathcal{M}_{1}\left(t_{0}\right)+B^{2}|\Omega|\left(t_{1}-t_{0}\right)\right] .
\end{array}
$$

Consequently the non-homogeneous version of (2.19)-(2.21) also holds and, when $b \equiv 0$ the non homogeneous version of (2.5) and (4.4) too. Moreover, $\mathcal{M}_{1}(t)$ is decreasing and right continuous, and if for some $T_{0}<T_{g} \leq T_{1}$

$$
\mathcal{M}_{1}\left(T_{1}\right)<\mathcal{M}_{1}\left(T_{0}\right)
$$

then, for any $R>0$ and $\tau>0$

$$
\int_{t_{0}}^{t_{1}} \int_{\Omega}\left(\sup _{S>R} \frac{1}{S^{\tau}} \int_{0}^{S} y^{\lambda / 2+1 / 2+\tau} f(t, x, y) d y\right)^{2} d x d t \geq C_{\tau}>0
$$

and

$$
\int_{T_{0}}^{T_{1}} \int_{\Omega}\left(\int_{e}^{\infty} \frac{y^{\lambda / 2+1 / 2}}{(\ln y)^{1 / 2}} f(t, x, y) d y\right)^{2} d x d t=+\infty
$$

We deduce from Theorem 8.1 the following gelation criteria.

Corollary 8.2 Consider the problem 8.1 under the conditions imposed above. Then gelation occurs for any weak solution with initial data satisfying

$$
\mathcal{M}_{1}(0) \geq C_{\lambda} B|\Omega|
$$

for some positive constant $C_{\lambda}$. If $b \equiv 0$, then for all weak solution with non identically zero initial data, gelation occurs and $\mathcal{M}_{1}(t)$ satisfies (1.18).

Sketch of the Proof. From the non homogeneous version of (2.21) with $k=1$ and the Cauchy Schwarz inequality we have

$$
\begin{aligned}
\frac{1}{|\Omega|} \int_{t_{1}}^{t_{0}} & \mathcal{M}_{1}^{2}(t) d t \leq \int_{t_{0}}^{t_{1}} \int_{\Omega}\left(M_{1}(t, x)\right)^{2} d x d t \leq \\
& \leq C_{1, \lambda}\left[\mathcal{M}_{1}\left(t_{0}\right)+\mathcal{M}_{0}\left(t_{0}\right)+B^{2}|\Omega|\left(t_{1}-t_{0}\right)\right] .
\end{aligned}
$$


The result for the general case $b \geq 0$ immediately follows from (8.7) and the condition (8.6) as in the proof of Theorem 1.4 in Section 6.

On the other hand, for the pure coagulation equation $(b \equiv 0)$ we first prove that $\mathcal{M}_{k}(t)$ is a decreasing function for $k=0$ and $k=1$, as in the proof of (2.9) in Section 4. Then we deduce from that fact, from the non homogeneous version of (1.16):

$$
\left.\forall T \geq 0 \quad \int_{T}^{\infty} \int_{\Omega}\left(M_{\lambda / 2}(t, x)\right)^{2} d x d t \leq 2 \mathcal{M}_{0} / T\right)
$$

and the non homogeneous version of (2.5),

$$
\forall T \geq 0 \quad \int_{T}^{\infty} \int_{\Omega}\left(\int_{R}^{\infty} f(t, x, y) y d y\right)^{2} d x d t \leq C_{\lambda} R^{1-\lambda} \mathcal{M}_{1}(T),
$$

the following non homogeneous version of (4.4)

$$
\forall T \geq 0 \quad \int_{T}^{\infty} \int_{\Omega}\left(M_{1}(t, x)\right)^{2} d x d t \leq C_{\lambda} \mathcal{M}_{0}^{\lambda-1}(0) \mathcal{M}_{1}(T)^{2-\lambda} .
$$

By the Cauchy Schwarz inequality again we obtain

$$
\frac{1}{|\Omega|} \int_{T}^{\infty} \mathcal{M} 1^{2}(t) d t \leq C_{\lambda} \mathcal{M}_{0}^{\lambda-1}(0) \mathcal{M}_{1}(T)^{2-\lambda}
$$

and we conclude that $\mathcal{M}_{1}(t)$ satisfies (1.18) thanks to Lemma 4.1.

Remark 8.3 Notice that also in this nonhomogeneous setting the only power like self similar behaviour in the $y$ variable of the solution $f$ compatible with the estimates (8.4, (8.5) and with the non homogeneous version of (2.20) and (2.21) is again $y^{-3 / 2-\lambda / 2}$.

Acknowledgment. We thank P. Laurençot for many useful discussions during the preparation of this work and for quote additional references. We were partially supported by CNRS and UPV through a PIC between the Universidad del Pais Vasco and the Ecole Normale Superieure.

\section{References}

[1] M. Aizenman and T. Bak, Convergence to equilibrium in a system of reacting polymers, Comm. Math. Phys. 65 (1979), 203-230.

[2] D.J. Aldous, Deterministic and stochastic models for coalescence (aggregation, coagulation): a review of the mean-field theory for probabilists, Bernoulli 5 (1999), 3-48. 
[3] J.M. Ball and J. Carr, The discrete coagulation-fragmentation equations : existence, uniqueness, and density conservation, J. Statist. Phys. 61 (1990), 203-234.

[4] E. Buffet and J.V. Pulé, Gelation: the diagonal case revisited, Nonlinearity 2 (1989), 373-381.

[5] A.V. Burobin and V.A. Galkin, Solutions of an equation of coagulation, Differential Equations 17 (1981), 456-462.

[6] J. Carr and F.P. da Costa, Instantaneous gelation in coagulation dynamics, Z. Angew. Math. Phys. 43 (1992), 974-983.

[7] F.P. da Costa, Existence and uniqueness of density conserving solutions to the coagulation-fragmentation equations with strong fragmentation, J. Math. Anal. Appl. 192 (1995), 892-914.

[8] P.G.J. van Dongen, On the possible occurrence of instantaneous gelation in Smoluchowski's coagulation equation, J. Phys. A 20 (1987), 1889-1904.

[9] P.G.J. van Dongen and M.H. Ernst, On the occurrence of a gelation transition in Smoluchowski's coagulation equation, J. Stat. Phys., 44 (1986), 785-792.

[10] P.G.J. van Dongen and M.H. Ernst, Scaling solutions of Smoluchowski's coagulation equation, J. Stat. Phys., 50 1/2 (1988), 295-329.

[11] R.L. Drake, A general mathematical survey of the coagulation, in: G. Hidy, J.R. Brocks (Eds.), Topics in Current Aerosol Research Reserach 3 (Part 2), Pergamon Press, Oxford (1972).

[12] P.B. Dubovskii, Mathematical theory of coagulation, Lecture Notes Ser. 23 Seoul Nat. Univ., Seoul (1994).

[13] P.B. Dubovskii and I.W. Stewart, Existence, uniqueness and mass conservation for the coagulation-fragmentation equation, Math. Methods Appl. Sci. 19 (1996), 571-591.

[14] M.H. Ernst, R.M. Ziff and E.M. Hendriks, Coagulation processes with a phase transition, J. Colloid Interface Sci. 97 (1984), 266-277.

[15] M. Escobedo, P. Laurençot, S. Mischler and B. Perthame, CoagulationFragmentation equations with strong fragmentation, work in preparation.

[16] V.A. Galkin, The existence and uniqueness of the solution of the coagulation equation, Differential equation 13 (1977), 1014-1021.

[17] V.A. Galkin, P.B. Dubovskii, Solution of the coagulation-fragmentation equations with unbounded kernels, Differential equation 22 (1986), 373378. 
[18] E.M. Hendriks, M.H. Ernst, R.M. Ziff, Coagulation equations with Gelation, Journal Stat. Phys. 33 (1983), 519-563.

[19] M.A. Herrero, J.J.L. Velazquez, D. Wrzosek, Sol-gel transition in a coagulation-diffuse model, Phys. D 141 (2000), 221-247.

[20] I. Jeon, Existence of gelling solutions for coagulation-fragmentation equations, Comm. Math. Phys. 194 (1998), 541-567.

[21] N.J. Kokholm, On Smoluchowski's coagulation equation, J.Phys. A: Math. Gen. 21 (1988), 839-842.

[22] Ph. Laurençot, The discrete coagulation equations : existence of solutions and gelation, in Journées Elie Cartan 1998 et 1999, Publications de l'Institut Elie Cartan 16, Institut Elie Cartan, Nancy, 2000, pp. 74-104.

[23] Ph. Laurençot, On a class of continuous coagulation-fragmentation models, J. Differential Equations 167 (2000), 145-174.

[24] Ph. Laurençot and S. Mischler, Global existence for the discrete diffusive Coagulation-Fragmentation equation in $L^{1}$, preprint of the E.N.S. Paris (2001).

[25] Ph. Laurençot and S. Mischler, The continuous Coagulation-Fragmentation equation with diffusion, preprint of the E.N.S. Paris (2001).

[26] Ph. Laurençot and S. Mischler, From discrete to continuous coagulationfragmentation models, preprint of the E.N.S. Paris (2001).

[27] F. Leyvraz, Existence and properties of post-gel solutions for the kinetic equations of coagulation, J. Phys. A: Math. Gen. 16 (1983), 2861-2873.

[28] F. Leyvraz and H.R. Tschudi, Singularities in the kinetics of coagulation processes, J. Phys. A: Math. Gen. 14 (1983), 3389-3405.

[29] J.B. McLeod, On an infinite set of non-linear differential equations I\& II, Q. J. Math. Oxf. Ser., 13 (1962), 119-128 \& 193-205.

[30] J.B. McLeod, On the scalar transport equation, Proc. London Math. Soc. 314 (1964), 445-458.

[31] Z.A. Melzak, A scalar transport equation, Trans. Amer. Math. Soc. 85 (1957), 547-560.

[32] H. Muller, Zur allgemeinen Theorie der raschen Koagulation, Kolloidchemische 27 (1928), 223-250.

[33] J.R. Norris, Cluster Coagulation, Commun. Math. Phys. 209 (2000), 407435.

[34] M. von Smoluchowski, Z. Phys. 96 (1916), 557-585. 
[35] M. Shirvani, H.J. van Roessel, Some results in the coagulation equation, Nonlinear Analysis 43 (2001), 563-573.

[36] J. Spouge, An existence theorem for the discrete coagulation-fragmentation equations, Math. Proc. Cambridge Philos. Soc. 96 (1984), 351-357.

[37] G. Stell and R.M. Ziff, Kinetics of polymer gelation, J. Chem. Phys. 73 (1980), 3492-3499.

[38] I.W. Stewart, A global existence theorem for the general coagulationfragmentation equation with unbounded kernels, Math. Methods Appl. Sci. 11 (1989), 627-648.

[39] I.W. Stewart, An uniqueness theorem for the coagulation-fragmentation equation, Math. Proc. Cambridge Philos. Soc. 107 (1990), 573-578.

[40] I.W. Stewart, On the coagulation-fragmentation equation, J. Appl. Math. Phys. (ZAMP) 41 (1990), 917-924.

[41] I.W. Stewart, Density conservation for a coagulation equation, Z. Angew. Math. Phys. 42 (1991), 746-756.

[42] W.H. Stockmayer, , J. Chem. Phys. 11 (1943), 45.

[43] W.H. White, A global existence theorem for Smoluchovski's coagulation equations, Proc. Am. Math. Soc., 80 (1980). 